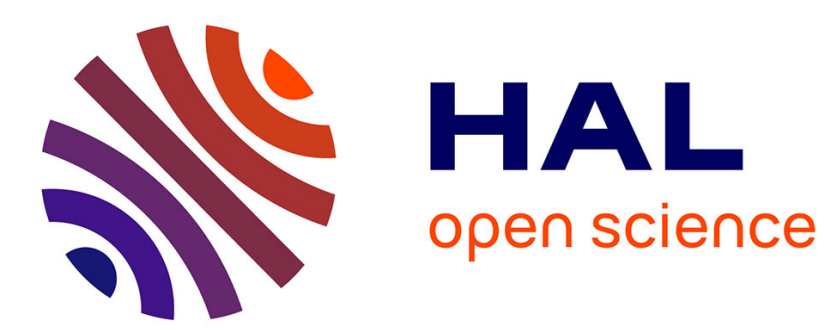

\title{
Comparison of three scattering models for ultrasound blood characterization
}

\author{
Emilie Franceschini, Ratan K Saha, Guy Cloutier
}

\section{To cite this version:}

Emilie Franceschini, Ratan K Saha, Guy Cloutier. Comparison of three scattering models for ultrasound blood characterization. IEEE Transactions on Ultrasonics, Ferroelectrics and Frequency Control, 2013, 60 (11), pp.2321-2334. 10.1109/TUFFC.2013.6644736 . hal-00908520

\section{HAL Id: hal-00908520 \\ https://hal.science/hal-00908520}

Submitted on 24 Nov 2013

HAL is a multi-disciplinary open access archive for the deposit and dissemination of scientific research documents, whether they are published or not. The documents may come from teaching and research institutions in France or abroad, or from public or private research centers.
L'archive ouverte pluridisciplinaire HAL, est destinée au dépôt et à la diffusion de documents scientifiques de niveau recherche, publiés ou non, émanant des établissements d'enseignement et de recherche français ou étrangers, des laboratoires publics ou privés. 


\title{
Comparison of three scattering models for ultrasound blood characterization
}

\author{
Emilie Franceschini, Member IEEE, Ratan K. Saha, and Guy Cloutier, Senior Member IEEE,
}

\begin{abstract}
Ultrasonic backscattered signals from blood contain frequency-dependent information that can be used to obtain quantitative parameters reflecting the aggregation level of red blood cells (RBCs). The approach consists in estimating structural aggregate parameters by fitting the spectrum of the backscattered radio-frequency echoes from blood to an estimated spectrum considering a theoretical scattering model. In this study, three scattering models were examined: a new implementation of the Gaussian Model (GM), the Structure Factor Size Estimator (SFSE) and the new Effective Medium Theory combined with the Structure Factor Model (EMTSFM). The accuracy of the three scattering models in determining mean aggregate size and compactness was compared by two- and three-dimensional (2D and 3D) computer simulations where RBC structural parameters are controlled. Two clustering conditions were studied: (1) when the aggregate size varied and the aggregate compactness was fixed in both $2 \mathrm{D}$ and $3 \mathrm{D}$ cases, and (2) when the aggregate size was fixed and the aggregate compactness varied in the $2 \mathrm{D}$ case. For both clustering conditions, the EMTSFM was found more suitable than the GM and SFSE for characterizing RBC aggregation.
\end{abstract}

E. Franceschini is with the Laboratoire de Mécanique et d'Acoustique LMA - CNRS UPR 7051, Aix-Marseille University, Centrale Marseille, F-13402 Marseille Cedex 20, France (e-mail: franceschini@1ma.cnrs-mrs.fr).

R. K. Saha is with the Saha Institute of Nuclear Physics, Applied Material Science Division, 1/AF Bidhannagar, Kolkata-700 064, India.

G. Cloutier is with the Laboratory of Biorheology and Medical Ultrasonics, University of Montreal Hospital Research Centre (CRCHUM), 2099 Alexandre de Sève (Room Y-1619), Montreal, Quebec H2L 2W5, Canada and with the Department of Radiology, Radio-Oncology and Nuclear Medicine, and Institute of Biomedical Engineering, University of Montreal, Montreal, Quebec, Canada. 


\section{Comparison of three scattering models for ultrasound blood characterization}

\section{INTRODUCTION}

Quantitative ultrasound (US) techniques are mainly based on the frequency analysis of backscattered signals by biological tissues to determine physical properties of the average tissue microstructure. These techniques rely on theoretical scattering models to fit the spectrum of backscattered echoes to an estimated spectrum using an appropriate model. The theoretical scattering model most frequently used for this purpose is the Gaussian model (GM) [1] [2] that yields two tissue properties: the average scatterer size and the acoustic concentration (i.e., the product of the scatterer number density by the square of the relative impedance difference between scatterers and the surrounding medium). This approach was used to characterize dilute scattering media such as the eye [3], prostate [4] and breast [5]. Blood has also been studied with this technique [6], although estimations could be biased.

An important contribution of ultrasonic blood characterization is to assess the level of red blood cell (RBC) aggregation, which is a surrogate marker of inflammation [7]. It is well known that when RBCs are under low shear rates $\left(<10 \mathrm{~s}^{-1}\right)$, they interact strongly with each other and form complex rouleaux or three-dimensional (3D) structures. When the shear rate increases, these rouleaux or compact structures disaggregate. The aggregation phenomenon in human blood is normal, however hyperaggregation is a pathological state associated with several circulatory diseases, such as deep venous thrombosis, atherosclerosis and diabetes mellitus. Blood characterization using US backscatter techniques provides the unique opportunity of monitoring RBC aggregation non-invasively and in vivo within blood vessels. This quantification may help to elucidate the role of RBC aggregation in the etiology of such diseases.

US backscatter by blood is mainly due to RBCs that constitute the vast majority (97\%) of the blood cellular content. Blood can thus be described as a biphasic fluid composed of RBCs immersed in plasma. Since RBCs are acoustically considered as weak scatterers (impedance contrast between RBCs and plasma being around 13\%), multiple scattering can be neglected. However, for such tissue, it is not straightforward to develop a theoretical scattering model because of the high density of RBCs (their volume fraction or hematocrit varies between 30 and 50\%) and their ability to form aggregates. The Structure Factor Model (SFM) [8] [9] is an US scattering model proposed to simulate the backscatter coefficient (BSC) of aggregated RBCs. The SFM sums the acoustic contributions from individual RBCs and models their interaction by a statistical mechanics structure factor, which is by definition the Fourier transform of the spatial distribution of RBCs [8] [9]. However, the SFM can hardly be implemented to estimate the structural aggregate parameters in the framework of an inverse problem formulation because of the intensive computational time to assess the structure factor by realizing distributions of aggregating RBCs. That is why Yu and Cloutier [10] developed the SFSE scattering theory that approximates the SFM by 
using a second-order Taylor expansion of the structure factor. The SFSE is thus not as accurate as the SFM. The SFSE estimates two physical parameters describing the microstructure of RBC aggregates: the packing factor that increases with erythrocyte clustering and the average aggregate isotropic radius. However, experiments with pig blood in controlled flow devices [10] and 3D numerical simulations of isotropic monodisperse aggregates [11] recently showed that the two indices are correlated and follow a quadratic relationship, thus reducing the BSC parameterization to one structural index.

Another scattering model called the Effective Medium Theory combined with the SFM (EMTSFM) was recently proposed to better approximate the SFM [12]. It assumes that aggregates of RBCs can be treated as individual homogeneous scatterers, which have effective properties determined by the acoustical characteristics and concentration of RBCs within aggregates. The EMTSFM allows characterizing the radius, and for the first time in the quantitative US field, the compactness of RBC aggregates [12]. In the field of clinical hemorheology [13], assessing the compactness of RBC aggregates is of high clinical importance since it is related to the binding energy between cells. Normal RBC aggregates form rouleaux type structures, whereas pathologies associated with stronger binding energies result in clumps of RBCs (close to a spherical isotropic packing) [14] [15].

In our previous study [12], the EMTSFM and the SFM were compared in the framework of a forward problem study to determine the BSC from a known distribution of RBCs with known acoustical parameters. The goodness of the approximation of the EMTSFM in comparison with the SFM was examined as a function of frequency and structural aggregate parameters (aggregate size and compactness). Based on a two-dimensional (2D) simulation study, the EMTSFM was found to approximate the SFM with relative errors less than $30 \%$ for a product of the wavenumber times the mean aggregate radius $k r_{a g} \leq 1.32$ [12]. The aims of the present paper are :

1) to evaluate the EMTSFM in an inverse problem framework, i.e. to determine RBC structural features from the measured BSC, and

2) to compare the EMTSFM with two other scattering models: the SFSE and a new implementation of the GM slightly modified to treat aggregating scatterers.

To our knowledge, there is no means to experimentally assess aggregate sizes at a normal physiological hematocrit of $40 \%$ because RBCs at that hematocrit are opaque to light. It would thus not be feasible to quantitatively evaluate the performance of the different models with real experimental data. In the field of blood imaging and characterization, the assessment of accuracy of a scattering model was only performed at a low hematocrit of $6 \%$ by comparing optical and acoustic measurements of RBC aggregate sizes [10]. In the current paper, we thus aim to determine the performance of three theoretical scattering models (the new implementation of the GM, the SFSE and the EMTSFM) to extract the aggregation parameters from computer simulations where RBC structural parameters (such as the hematocrit, the aggregate size and compactness) are known.

The important contribution of the EMTSFM is the parameterization of the BSC with the aggregate compactness [12], which is a structural parameter not available in any other modeling strategies proposed in quantitative US. The potential of the EMTSFM and of the two other scattering models in estimating the aggregate compactness was examined by 2D computer simulations based on the SFM in controlled clustering configurations (when the 
aggregate compactness varies and the aggregate radius is fixed). This clustering condition was only conducted in $2 \mathrm{D}$ because of the computational load required to generate three-dimensional RBC distributions with various aggregate compactnesses with the SFM [12]. Some 3D computer simulations were also used in the controlled clustering configurations as those performed in $2 \mathrm{D}$ (when the aggregate size vary and the aggregate compactness is fixed) in order to compare the BSC behavior between 2D and 3D simulations, and estimated structural aggregate parameters with the three scattering models.

\section{Computer simulations based on the Structure Factor Model (SFM)}

This section presents computer simulations performed to predict the frequency dependence of the BSC from aggregated RBCs based on the SFM. In the following, it is assumed that the incident wavelength $\lambda$ is large compared to the RBC size. Consequently, the RBC shape could be approximated by a simple geometry having an equivalent surface in $2 \mathrm{D}$ or having an equivalent volume of a $\mathrm{RBC}$ in $3 \mathrm{D}$ [16]. RBCs were modeled as parallel infinite cylinders in the $2 \mathrm{D}$ case and as spheres in the $3 \mathrm{D}$ case of radius $a$, that have small contrast in acoustical properties relatively to the plasma (see Table I). This RBC shape approximation has some limitations for larger frequencies $(>20 \mathrm{MHz})$ and will be discussed later in Section V-C.

The SFM describing US backscatter by biological tissues consists of summing contributions from cells and modeling the cellular interaction by a statistical mechanics structure factor [8] [9]. By considering a collection of $N$ identical and weak scattering RBCs, the BSC expression can be written as:

$$
B S C_{S F M}(-2 k)=m \sigma_{b}(-2 k) S(-2 k),
$$

where $k$ is the wavenumber and $m$ the number density of RBCs that is related to the systemic hematocrit $\phi$ as $m=\phi / A_{p}$ (where $A_{p}$ is the RBC area) for $2 \mathrm{D}$ modeling, or as $m=\phi / V_{p}$ (where $V_{p}$ is the RBC volume) for $3 \mathrm{D}$ modeling. The backscattering cross section $\sigma_{b}$ of a single weak RBC was calculated using the fluid infinite cylinder expression in the 2D case [12] or using the fluid-filled sphere expression in the 3D case [17] [18] given by:

$$
\begin{aligned}
\sigma_{b}(-2 k) & =\frac{k^{3} A_{p}^{2} \gamma_{z}^{2}}{2 \pi}\left(\frac{J_{1}(2 k a)}{k a}\right)^{2} \quad \text { in the 2D case } \\
& =\frac{k^{4} V_{p}^{2} \gamma_{z}^{2}}{4 \pi^{2}}\left(3 \frac{\sin (2 k a)-2 k a \cos (2 k a)}{(2 k a)^{3}}\right)^{2} \quad \text { in the 3D case }
\end{aligned}
$$

where $J_{1}$ is the first order Bessel function of the first kind and $\gamma_{z}$ is the relative impedance difference between the RBC and its suspending medium (i.e., the plasma). The function $S$ is the structure factor representing the spatial positioning of RBCs and is defined by:

$$
S(-2 k)=E\left[\frac{1}{N}\left|\sum_{i=1}^{N} e^{-i 2 \mathbf{k r}_{i}}\right|^{2}\right]
$$

where $E$ represents the expected value of a random variable and $\mathbf{r}_{i}$ the position vectors defining the center of the $i$ th RBC in space. In general, the structure factor of a medium containing RBCs distributed in the 2D space (or in the 3D space) can be determined from the 2D Fourier transform (or 3D Fourier transform) of the spatial distribution 
of particles (see the Appendix of Ref. [19]).

The computation of the $B S C_{S F M}$ using the SFM requires an intensive computation because of the calculation of the structure factor $S$ as described in Eq. (1). Since the structure factor is by definition a statistical quantity, an average of structure factors from several RBC spatial distributions can give an estimated value of $S$. Because of the computational load to generate aggregating RBC distributions, a simple and fast method was used to randomly generate non-overlapping RBC aggregates which were isotropic and similar in size. For the 2D and the 3D computer simulations, the simulated $B S C_{S F M}$ were obtained from the method described in section III of Refs. [12] and [11], respectively. Note that 2D simulations are computationally less expensive but significant insights can be gained by studying $2 \mathrm{D}$ systems. On the other hand, 3D simulations are intuitively appealing because they better mimic experimental situations but are computationally expensive. These methods are briefly summarized in the following.

Random distributions for aggregating RBCs were computed within the simulated surface area of $600 \times 600 \mu \mathrm{m}^{2}$ in the $2 \mathrm{D}$ case and within the simulated volume of $1000 \times 125 \times 125 \mu m^{3}$ in the $3 \mathrm{D}$ case. The RBC radius $a$ was set to $2.75 \mu \mathrm{m}$ for all simulations. We first specified the systemic hematocrit $\phi$, the aggregate radius $r_{a g}$ and the aggregate compactness $\phi_{i}$ (i.e. the RBC concentration within aggregate). Aggregates of identical radii $r_{a g}$ and of identical compactness $\phi_{i}$ were then randomly distributed with non-overlapping positions to give the desired concentration of aggregates $\phi_{a g}=\phi / \phi_{i}$. Note that in the case of the 3D study, a small number of non-aggregated RBCs was added to reach the desired systemic hematocrit. It means that all the RBCs were aggregated in blood in the $2 \mathrm{D}$ case, whereas a fraction of RBCs were aggregated while the rest remained disaggregated in the 3D case. Finally, RBC distributions within aggregates were then generated as follows:

- in the $2 \mathrm{D}$ case, the locations of the RBCs were generated using external and repulsive forces to obtain random RBC positions inside each aggregate, such that the distribution of RBCs within each aggregate was different [12]. This technique allowed to study several aggregation configurations (1) when the aggregate size varied and the aggregate compactness was fixed to 0.6 , and (2) when the aggregate compactness varied from 0.3 to 0.6 and the aggregate size was fixed.

- in the 3D case, the RBCs were stacked by following a hexagonal close packing (HCP) structure for each aggregate, such that the distribution of RBCs within each aggregate was identical. This HCP structure provides the highest compactness that is about 0.74 for spheres [11]. Therefore, this technique allowed to study several aggregation configurations when the aggregate size varied and the aggregate compactness was fixed to 0.74 .

For each distribution of RBCs, the 2D or 3D Fourier transformation of the spatial organization of RBCs was then computed to obtain the corresponding structure factor. A mean structure factor was determined from 400 different tissue realizations in the 2D case (see section III.B in Ref. [12]) and from 250 different tissue realizations in the 3D case (see section III.B in Ref. [11]). 


\section{ULTRASOUND BACKSCATTERING MODELING FOR THE ESTIMATION OF STRUCTURAL AGGREGATE}

\section{PARAMETERS}

As seen previously in section II, the SFM allows to simulate the BSC from RBCs whatever the RBC spatial distribution (i.e. disaggregated or aggregated RBCs and/or with various aggregate sizes and compactnesses). However, the SFM can hardly be implemented to estimate structural parameters in the framework of an inverse problem formulation because of the intensive computational time to assess the structure factor by realizing distributions of RBCs with simulations. That is why two scattering theories, named the SFSE and the EMTSFM, have been recently developed to approximate the SFM for practical assessments of RBC structural features (i.e., in an inverse problem formulation). This section presents these two scattering theories (the SFSE and the EMTSFM) as well as the GM also used for tissue characterization. In this work, we present a new implementation of the GM model inspired by our development on EMTSFM. All three theories fit a curve to the simulated $B S C_{S F M}$ from blood to estimate aggregation parameters using the minimization routine "fminsearch" in MATLAB (The MathWorks, Inc., Natick, MA), i.e. a Nelder-Mead simplex method. Note that this fit was realized in the frequency bandwidth from $4 \mathrm{MHz}$ to the frequency corresponding to the first minimum of the $B S C_{S F M}$ (i.e., after the frequency-dependent increase in BSC followed by a peak and a reduction to its first minimum).

\section{A. The Structure Factor Size Estimator (SFSE)}

The SFSE developed by Yu and Cloutier [10] approximates the SFM with a second-order Taylor expansion of the structure factor in $k$ as follows:

$$
\begin{aligned}
S(-2 k) & \approx W-4\left(k R_{g} a\right)^{2} \quad \text { in the } 2 \mathrm{D} \text { case } \\
& \approx W-4\left(k R_{g} a\right)^{2} \approx W-\frac{12}{5}\left(k R_{s p} a\right)^{2} \quad \text { in the 3D case }
\end{aligned}
$$

where $W$ is the low-frequency limit of the structure factor $\left(\left.S(k)\right|_{k \rightarrow 0}\right)$ called the packing factor [20] [21] and $R_{g}$ is the radius of gyration of RBC aggregates assumed to be isotropic and expressed in number of RBCs [10]. Note that in the 3D case, $R_{g}$ is related to the isotropic radius $R_{s p}$ of an aggregate (expressed in number of RBCs) by $R_{g}=\sqrt{\frac{3}{5}} R_{s p}$ [11] [22]. By assuming identical RBCs, and recombining Eqs. (1) and (4), the SFSE model approximates the BSC as follows:

$$
\begin{aligned}
B S C_{S F S E}(-2 k) & =\frac{1}{2 \pi} m k^{3} A_{p}^{2} \gamma_{z}^{2}\left(\frac{J_{1}(2 k a)}{k a}\right)^{2}\left(W-4\left(k R_{g} a\right)^{2}\right) \quad \text { in the } 2 \mathrm{D} \text { case } \\
& =\frac{1}{4 \pi^{2}} m k^{4} V_{p}^{2} \gamma_{z}^{2}\left(3 \frac{\sin (2 k a)-2 k a \cos (2 k a)}{(2 k a)^{3}}\right)^{2}\left(W-\frac{12}{5}\left(k R_{s p} a\right)^{2}\right) \quad \text { in the 3D case }
\end{aligned}
$$

The SFSE assumes that the hematocrit $\phi$, the RBC radius $a$ and the acoustical properties of plasma and RBCs are known a priori. Therefore, Eq. (5) presents only two unknowns that characterize the aggregate structure: $W$ and $R_{g}$ (or equivalently, $W$ and $R_{s p}$ in the $3 \mathrm{D}$ case). Estimated values of $W^{*}$ and $R_{g}^{*}$ (or equivalently, $W^{*}$ and $R_{s p}^{*}$ in the $3 \mathrm{D}$ case) were determined by fitting the simulated $B S C_{S F M}$ given by Eq. (1) with $B S C_{S F S E}$ given by Eq. (5). 


\section{B. The Effective Medium Theory Combined with the Structure Factor Model (EMTSFM)}

The EMTSFM assumes that all the scatterers are aggregated, that the aggregates are identical and isotropic and that the scatterers within aggregates are evenly distributed [12]. In the case of blood backscatter, the model consists of treating the RBC aggregates as individual homogeneous particles of radius $r_{a g}$. These homogeneous particles are characterized by a density $\rho_{a g}$ and a compressibility $\kappa_{a g}$ derived from the acoustical properties of the two fluids constituting them (i.e., $\rho_{1}, \rho_{2}, \kappa_{1}$ and $\kappa_{2}$, where 1 indicates properties of RBCs and 2 those of plasma), and from the internal concentration of RBCs within the aggregates, defined as the aggregate compactness $\phi_{i}$, as follows:

$$
\begin{aligned}
\rho_{a g} & =\phi_{i} \rho_{1}+\left(1-\phi_{i}\right) \rho_{2} \\
\frac{1}{\kappa_{a g}} & =\frac{\phi_{i}}{\kappa_{1}}+\frac{1-\phi_{i}}{\kappa_{2}}
\end{aligned}
$$

The BSC from blood is then obtained by summing contributions from individual effective particles of radius $r_{a g}$ and modeling the effective particle interaction by a statistical mechanics structure factor $S_{a g}$. The equivalent BSC expression is thus given by [12]:

$$
B C_{E M T S F M}(-2 k)=m_{a g} \sigma_{a g}(-2 k) S_{a g}(-2 k),
$$

where $S_{a g}$ is the structure factor of a collection of $N_{a g}$ identical particles of radius $r_{a g}$ randomly distributed and $m_{a g}$ is the number density of aggregates that is related to the effective aggregate concentration $\phi_{a g}$. The effective aggregate concentration is equal to the RBC concentration in blood $\phi$ divided by the aggregate compactness $\phi_{i}$ : $\phi_{a g}=\phi / \phi_{i}$. The backscatter cross-section of an effective single particle $\sigma_{a g}$ was calculated using the fluid infinite cylinder expression in the $2 \mathrm{D}$ case [12] or using the fluid-filled sphere expression in the 3D case [17] [18] given by:

$$
\begin{aligned}
\sigma_{a g}(-2 k) & =\frac{k^{3} \pi r_{a g}^{4} \gamma_{z_{a g}}^{2}}{2}\left(\frac{J_{1}\left(2 k r_{a g}\right)}{k r_{a g}}\right)^{2} \quad \text { in the 2D case } \\
& =\frac{4 k^{4} r_{a g}^{6} \gamma_{z_{a g}}^{2}}{9}\left(3 \frac{\sin \left(2 k r_{a g}\right)-2 k r_{a g} \cos \left(2 k r_{a g}\right)}{\left(2 k r_{a g}\right)^{3}}\right)^{2} \quad \text { in the 3D case }
\end{aligned}
$$

where $z_{a g}$ is the impedance of the equivalent particle and $\gamma_{z_{a g}}$ the relative impedance difference between the equivalent particle and the plasma $\left(\gamma_{z_{a g}}=\left(z_{a g}-z_{2}\right) / z_{2}\right)$. For a random distribution of hard-cylinders in 2D, the structure factor was numerically computed as described in the appendix A. For a random distribution of hard-spheres in 3D, the structure factor can be analytically calculated as established by Wertheim [23]. The analytical expression for the structure factor in the $3 \mathrm{D}$ case is described in the appendix $\mathrm{B}$.

By assuming that the hematocrit $\phi$, the RBC radius $a$ and the acoustical properties of plasma and RBCs are known a priori, the unknown parameters are the radius of aggregates $r_{a g}$ and their compactness $\phi_{i}$. The unknown parameters were estimated by matching the simulated $B S C_{S F M}$ given by Eq. (1) with the theoretical $B S C_{E M T S F M}$ given by Eq. (7).

\section{The Gaussian Model (GM)}

Using the GM, the BSC is modeled with a spatial autocorrelation function describing the shape and distribution of scatterers in the medium. The scattering sites are assumed to be randomly distributed and of simple geometric 
shapes, represented as gaussian scatterers mimicking continuous changes in impedance. In this framework, the BSC can be written as the product of the theoretical BSC under Rayleigh scattering and the backscatter form factor (see Eqs. (74)-(76) of Ref. [18] for the GM formulation in 3D). The form factor describes the frequency dependence of BSC attributed to the size and shape of the prototype scatterer. The Gaussian form factor has been used for many applications [3]-[6]. It represents tissue structures as continuously varying distributions of acoustic impedance fluctuations about the mean value, and the effective radius is related to the impedance distribution of the scatterers.

The BSC for the GM formulation is written as the product of the BSC in the Rayleigh limit and the backscatter form factor as follows [18]:

$$
\begin{aligned}
B S C_{G M}(-2 k) & =\frac{k^{3} S_{s}^{2} n_{z}}{2 \pi} e^{-2 k^{2} d^{2}}=\frac{\pi k^{3} a_{e f f}^{4} n_{z}}{2} e^{-k^{2} a_{\text {eff }}^{2}} \quad \text { in the 2D case } \\
& =\frac{k^{4} V_{s}^{2} n_{z}}{4 \pi^{2}} e^{-2 k^{2} d^{2}}=\frac{4 k^{4} a_{e f f}^{6} n_{z}}{9} e^{-0.827 k^{2} a_{\text {eff }}^{2}} \quad \text { in the 3D case }
\end{aligned}
$$

where $n_{z}$ is the acoustic concentration (i.e., the product of the number density of scatterers times the square of the relative impedance difference $\gamma_{z}$ between scatterers and the surrounding tissue). In the 2D case (or respectively in the $3 \mathrm{D}$ case), the characteristic dimension $d$ is related to the area of the effective scatterer $S_{s}$ by: $S_{s}=2 \pi d^{2}$ (or related to the volume of the effective scatterer $V_{s}$ by: $V_{s}=\left(2 \pi d^{2}\right)^{3 / 2}$ ). Continuous isotropic media can be characterized by the correlation distance $d$, in the same way as discrete isotropic media are characterized by a scatterer radius [18]. The effective radius of the scatterer $a_{e f f}$ is related to the correlation distance $d$ by setting values of $S_{s}$ (or $V_{s}$, respectively) for a continuum model equal to the area of an effective cylinder (or equal to the volume of an effective scatterer) of radius $a_{e f f}: S_{s}=2 \pi d^{2}=\pi a_{e f f}^{2}$ or $V_{s}=\left(2 \pi d^{2}\right)^{3 / 2}=(4 / 3) \pi a_{e f f}^{3}$.

Estimates of the effective radius $a_{e f f}^{*}$ and acoustic concentration $n_{z}^{*}$ were determined by fitting the simulated $B S C_{S F M}$ given by Eq. (1) with $B S C_{G M}$ given by Eq. (9). Effective radii $a_{e f f}$ estimated with the GM have been hypothesized to be related to the aggregate radii, and the acoustic concentration $n_{z}$ is postulated to be the product of the number density of aggregates times the square of the relative impedance difference between aggregates and the plasma as follows:

$$
\begin{aligned}
n_{z} & =\gamma_{z_{a g}}^{2} \frac{\phi_{a g}}{\pi a_{e f f}^{2}}=\left(\frac{z_{a g}-z_{2}}{z_{2}}\right)^{2} \frac{\phi}{\phi_{i} \pi a_{e f f}^{2}}, \quad \text { in the 2D case } \\
& =\gamma_{z_{a g}}^{2} \frac{3 \phi_{a g}}{4 \pi a_{e f f}^{3}}=\left(\frac{z_{a g}-z_{2}}{z_{2}}\right)^{2} \frac{3 \phi}{4 \phi_{i} \pi a_{e f f}^{3}}, \quad \text { in the 3D case }
\end{aligned}
$$

where $z_{a g}$ is the effective impedance of the aggregates approximated by the mixing law: $z_{a g}=\phi_{i} z_{1}+\left(1-\phi_{i}\right) z_{2}$. Since the hematocrit $\phi$ and the acoustical properties of plasma and RBCs are assumed to be known $a$ priori, the aggregate compactness can be deduced from the estimated parameters $a_{\text {eff }}^{*}$ and $n_{z}^{*}$ by using Eq. (10) as follows:

$$
\begin{aligned}
\phi_{i}^{*} & =\frac{\pi a_{e f f}^{*} n_{z}^{*} z_{2}^{2}}{\phi\left(z_{2}-z_{1}\right)^{2}} \quad \text { in the } 2 \mathrm{D} \text { case } \\
& =\frac{4 \pi a_{e f f}^{*}{ }^{3} n_{z}^{*} z_{2}^{2}}{3 \phi\left(z_{2}-z_{1}\right)^{2}} \quad \text { in the } 3 \mathrm{D} \text { case }
\end{aligned}
$$

It means that the new proposition of the GM was employed in our study as an effective medium model, but contrary to the EMTSFM, the GM is not combined with the SFM (such that the GM is assumed to be accurate only at low 
systemic hematocrits). In the following, we thus give the estimated parameters $a_{\text {eff }}^{*}$ and $\phi_{i}^{*}$ with the GM, instead of the classical estimated parameters $a_{e f f}^{*}$ and $n_{z}^{*}$.

\section{RESUlts}

This section gives the results of the inverse problem obtained for 2D and 3D computer simulations with the three backscattering models aforementioned: SFSE, EMTSFM and GM.

\section{A. Results obtained from the $2 D$ computer simulations}

For the 2D computer simulations, we first studied clustering configurations where the aggregate compactness was fixed to $\phi_{i}=60 \%$ and the aggregate radius $r_{a g} / a$ varied, and then clustering configurations where the aggregate radius was fixed to $r_{a g} / a=6.32$ and the aggregate compactness $\phi_{i}$ varied.

1) Results for the SFSE: The SFSE was first examined for systemic hematocrits of 10, 20 and $30 \%$ when the aggregate size varied and the aggregate compactness was fixed to a high value: $\phi_{i}=60 \%$. Figure 1 shows $B S C_{S F M}$ as a function of frequency for different aggregate sizes and systemic hematocrits. The symbols represent the $B S C_{S F M}$ computation for the disaggregated case $\left(r_{a g} / a=1\right)$ and for aggregation with radii $r_{a g} / a=3.16,5.0$ and 7.07. Also represented in dashed lines in Fig. 1 are corresponding $B S C_{S F S E}$ fitted curves. The first peaks of the simulated $B S C_{S F M}$ occur at lower frequencies as the aggregate radius increases. Since the fitting curves with the SFSE were realized in the frequency bandwidth from $4 \mathrm{MHz}$ to the frequency corresponding to the first minimum of the $B S C_{S F M}$ (except for the disaggregated case for which the frequency bandwidth is from 4 to 50 $\mathrm{MHz}$ ), the bandwidth used for the fitting becomes smaller as the aggregate radius increases. It is clear from the figure that the SFSE provided better fits for the lower hematocrit of $10 \%$. As the hematocrit increases, the SFSE model is insufficient to predict the behavior of $B S C_{S F M}$, especially in the low frequency range. The estimated values of $W^{*}$ and $R_{g}^{*}$ are given in Table II for systemic hematocrits of 10, 20 and 30\%. In this table, the relative error for parameter $R_{g}^{*}$ corresponds to: $\epsilon_{R_{g}^{*}}=\left(R_{g}^{*}-\left(r_{a g} / a\right)\right) /\left(r_{a g} / a\right)$. Figure $2 \mathrm{a}$ shows the estimated values of $R_{g}^{*}$ as a function of the actual aggregate radii $r_{a g} / a$ for all hematocrits. Also represented are the corresponding linear regression lines showing good correlation $r^{2} \geq 0.95$ at all hematocrits. For radii $r_{a g} / a$ between 4.47 and 7.95, relative errors $\epsilon_{R_{g}^{*}}$ were less than $30 \%$ for hematocrits of 10 and $20 \%$. It is interesting to notice that estimated parameters $W^{*}$ and $R_{g}^{*}$ follow a linear relation for all hematocrits (see Fig. 2b).

The SFSE was also evaluated at systemic hematocrits of 10 and $20 \%$ when the aggregate size $r_{a g} / a$ was fixed to 6.32 and the aggregate compactness $\phi_{i}$ varied from 30 to $60 \%$. It is important to emphasize that 2D random particle distributions could be easily generated using a random number generator up to an area fraction of approximatively 0.5. For the $20 \%$ systemic hematocrit, aggregate compactnesses smaller than $40 \%$ could not be computed because the corresponding area fractions of aggregates were too high: $\phi_{a g}>0.5$. Similarly, the variation of the aggregate compactness could not be performed at a systemic hematocrit of $30 \%$ because the area fractions of aggregates is already equal to 0.5 for an aggregate compactness $\phi_{i}=60 \%$. Figure 3 displays $B S C_{S F S E}$ in dashed lines for the following clustering conditions: $r_{a g} / a=6.32$ and $\phi_{i}$ varying from 30 to $60 \%$. One can notice large differences 
between simulated and fitted SFSE curves, especially at low frequencies where the fitted curves over-estimate the $B S C_{S F M}$ amplitude. These differences are larger at $\phi=20 \%$. The estimated values of $R_{g}^{*}$ for different aggregate compactnesses are plotted in Fig. 4a. Although the true radius is fixed, estimated $R_{g}^{*}$ increases with the aggregate compactness at both hematocrits. We found no correlation between the actual fixed radius and the estimated radii $\left(r^{2}<0.06\right)$. Notice the linear relation between $W^{*}$ and $R_{g}^{*}$ when the aggregate compactness varies (see Fig. 4b), as observed previously in Fig. $2 \mathrm{~b}$ when the aggregate radius $r_{a g} / a$ was changed.

2) Results for the EMTSFM: The BSC curves fitted with the EMTSFM are shown in solid lines in Fig. 1 for the case of varying values of $r_{a g} / a$, and in Fig. 3 for varying $\phi_{i}$. In both cases, the EMTSFM provided good fittings to the simulated $B S C_{S F M}$ curves for all systemic hematocrits. For the clustering conditions where the aggregate radius varied and the aggregate compactness was constant, the estimated values $r_{a g}^{*} / a$ and $\phi_{i}^{*}$, and corresponding relative errors are given in Fig. 5 for systemic hematocrits of 10, 20 and 30\%. For the clustering conditions where the aggregate compactness varied and the aggregate radius was constant, the results are shown in Fig. 6 for systemic hematocrits of 10 and 20\%. For the EMTSFM, the relative errors for each parameter correspond to:

$$
\epsilon_{r_{a g}^{*}}=\frac{\left(r_{a g}^{*} / a\right)-\left(r_{a g} / a\right)}{\left(r_{a g} / a\right)} \text { and } \epsilon_{\phi_{i}^{*}}=\frac{\phi_{i}^{*}-\phi_{i}}{\phi_{i}} \text {. }
$$

In both sub-studies where the aggregate radius and compactness varied, a very good correspondence can be observed between true and estimated aggregate sizes and compactnesses. The relative errors for the estimated aggregate radii and compactnesses were less than $13 \%$ and 14\%, respectively, for all hematocrits and for all studied aggregating configurations.

3) Results for the GM: Figure 7 presents the $B S C_{S F M}$ curves fitted with the GM for several aggregate sizes at the same clustering conditions as in Fig. 1. The GM provided over-estimates in the low frequency range for all systemic hematocrits. Excellent correlations $\left(r^{2} \geq 0.92\right)$ were found between the estimated and true aggregate radii for all hematocrits (data not shown). The estimated values $a_{e f f}^{*} / a$ and $\phi_{i}^{*}$ from the GM, and corresponding relative errors are given in Fig. 8. For systemic hematocrits of 10 and 20\%, the estimated radii and compactnesses are quantitatively satisfactory with relative errors less than $15 \%$. However, for $\phi=30 \%$, the relative errors increase up to $40 \%$.

For the clustering conditions where the aggregate compactness varied and the aggregate radius was constant, the results are shown in Fig. 9 for systemic hematocrits of 10 and 20\%. As previously observed with the SFSE, the estimated effective radius increases as the aggregate compactness increases. The estimated radii and compactnesses matched the true parameters at $\phi=10 \%$ with relative errors less than $17 \%$. However, for $\phi=20 \%$, large relative errors (up to $74 \%$ ) were obtained.

4) Comparison of the errors between the simulated BSC and the fitted curves with the three scattering models: The errors (i.e. differences) between the simulated BSC and the fitted curves with the three scattering models (SFSE, EMTSFM and GM) are presented in Fig. 10. The logarithm of the error is shown to enhance reading. The error reveals how the models fit the data. It is clear from the figure that errors were smaller with the EMTSFM and larger with the GM, for each hematocrit. For the aggregating conditions where the aggregate radius varied, the 
error decreases as the radius increases. When the aggregate radius increases, the bandwidth frequency used for the fit becomes smaller and therefore, the number of frequencies used for the error computation decreases.

\section{B. Results obtained from the $3 D$ computer simulations}

For the 3D computer simulations, the GM, SFSE and EMSTFM were examined when the aggregate size varied and the aggregate compactness was fixed to a high value: $\phi_{i}=74 \%$.

It is important to note that the 3D simulated aggregates were highly packed leaving small numbers of particles as non-aggregated RBCs. For each tissue realization, the actual mean aggregate radius $r_{a g}$ was computed using Eq. (6) in Ref. [11], and then the concentration of aggregated RBCs $\phi^{\prime}$ was computed as:

$$
\phi^{\prime}=\frac{\phi_{i} N_{a g}(4 / 3) \pi r_{a g}^{3}}{1000 \times 125 \times 125 \times\left(10^{-6}\right)^{3}} .
$$

Figure 11a shows the values of $\phi^{\prime}$ as a function of the mean aggregate radius $r_{a g} / a$ for the three systemic hematocrits of 20, 30 and 40\%. The percentage of disaggregated RBCs was between 20 and $30 \%$ for the systemic hematocrit of $20 \%$ and between 27 and $37 \%$ for the systemic hematocrit of $40 \%$. Note that the three models presented in section III assumed that all RBCs were aggregated in blood and that aggregates had identical shape and size. Consequently, during the inversion procedure of the 3D BSC data, we neglected the contribution of the disaggregated RBCs on the simulated $B S C_{S F M}$ and we replaced the hematocrit $\phi$ by the value of the concentration of aggregated RBCs $\phi^{\prime}$.

Figure $11 \mathrm{~b}$ and c shows $B S C_{S F M}$ as a function of frequency for several aggregate sizes and systemic hematocrits of 30 and $40 \%$. Also represented in Fig. 11b and c are corresponding fitted curves obtained with the SFSE, EMTSFM and GM. The fitted GM and SFSE curves did not produce good fits to the 3D data and overestimated the $B S C_{S F M}$ amplitude (especially in the low frequency range), as observed in the 2D case (see Figures 1 and 7). At the opposite, the EMTSFM provided good fittings to the simulated $B S C_{S F M}$ curves.

The results obtained with the SFSE were already presented in a previous article [11]. Excellent correlations $\left(r^{2} \geq 0.94\right)$ were found between the estimated and true aggregate radii for all hematocrits (see Fig. 5a in Ref. [11]). It can also be seen in Fig. 5a of Ref. [11] that for each hematocrit there is an aggregate size range where the SFSE method works at its best. For example, relative errors for estimated radii were less than $20 \%$ for true radius values between 14 and $17 \mu \mathrm{m}$ at the hematocrit of $40 \%$. The parameters $W^{*}$ and $R_{s p}^{*}$ followed a quadratic relationship (as in Fig. 5b of Ref. [11]).

Figure 12 gives the values of $r_{a g}^{*}$ and $\phi_{i}^{*}$ estimated with the EMTSFM and corresponding relative errors that were less than $15 \%$ and $23 \%$, respectively, for all hematocrits. Figure 13 gives the values of $a_{e f f}^{*}$ and $\phi_{i}^{*}$ estimated with the GM and corresponding relative errors. The estimated radii with the new formulation of the GM are quantitatively satisfactory with relative errors less than $9 \%$ for all hematocrits. The relative errors for the estimated compactnesses with the GM are larger with relative errors up to $32 \%$ for the hematocrits of 20 and $30 \%$, and up to $76 \%$ for the hematocrit of $40 \%$. 


\section{Discussion AND CONCLUSIONS}

Three scattering models for the characterization of RBC aggregation were examined. From these models, the gold standard simulated $B S C_{S F M}$ was fitted and aggregation parameters were extracted. The SFSE has been developed for blood characterization and the GM is a model that has been used in various tissue studies. Herein, the radius estimates $R_{g}$ from the SFSE and $a_{e f f}$ from the GM were hypothesized to represent the aggregate size.

A. Clustering conditions where the aggregate radius varied and the aggregate compactness was constant (2D and $3 D$ computer simulations)

The $2 \mathrm{D}$ and $3 \mathrm{D}$ computer simulations were performed on the same clustering configuration where the aggregate radius varied and the aggregate compactness was constant. It is interesting to observe the same $B S C_{S F M}$ behavior for both $2 \mathrm{D}$ and $3 \mathrm{D}$ studies. Indeed, the simulated $B S C_{S F M}$ amplitude increases with the size of aggregates and the $B S C_{S F M}$ first peaks occur at lower frequencies as the aggregate radius increases (see Fig. 1 in the $2 \mathrm{D}$ case, and Fig. 11b and c of the present paper and Fig. 4 of Ref. [11] in the 3D case). Moreover, as it can be observed in Figures 1 and 7 in the 2D case and in Figure 11 in the 3D case, the data fitting quality obtained with the three models were quite similar. In both 2D and 3D cases, it is clear that the GM and the SFSE are insufficient to model the complex behavior of BSC and that the EMTSFM was the model that better fitted the BSC data for all studied hematocrits.

Although the SFSE model did not produce good spectral fits to the BSC data for 2D and 3D computer simulations, significant correlations were found between the estimated and true radii with $r^{2}$ superior to 0.95 at all hematocrits (see Fig. 2a in the present paper and see Fig. 5a of Ref. [11]). However, the estimated aggregation parameters $W^{*}$ and $R_{g}^{*}$ followed a linear relationship in our 2D simulation study. This relation was also found to be quadratic in 3D numerical simulations [11] and under experimental conditions [10]. It means that the BSC parameterization can be reduced to one parameter and that no new information can be obtained with the parameter $W^{*}$.

The EMTSFM and the GM used as effective medium models gave quantitatively satisfactory radius estimates with relative errors less than $15 \%$ for the 10 and $20 \%$ hematocrits in the $2 \mathrm{D}$ case, and for all hematocrits in the $3 \mathrm{D}$ case. For the highest systemic hematocrit, the aggregate compactnesses were better estimated with the EMTSFM with relative errors less than $14 \%$ in the $2 \mathrm{D}$ case (and less than $23 \%$ in the $3 \mathrm{D}$ case), whereas the relative errors were between 19 and $36 \%$ in the 2D case (and between 59 and $76 \%$ in the 3D case) for the GM. These results with the EMTSFM and the GM were somewhat anticipated since the assumption of a random distribution of scatterers used by the GM fails due to the spatial correlation between scatterers in a dense medium [24]. To conclude, the EMTSFM was more suitable than the GM and SFSE for characterizing the aggregate microstructure in both 2D and $3 \mathrm{D}$ studies. 
B. Clustering conditions where the aggregate compactness varied and the aggregate radius was constant $(2 D$ computer simulations)

For the highest simulated hematocrit of $20 \%$, the aggregate radii normalized by the RBC radius were estimated between 4.34 and 8.55 using the SFSE model and between 3.83 and 5.33 using the GM (see Fig. 4a and Fig. 9), whereas the actual aggregate radius was $r_{a g} / a=6.32$. Therefore, we found no correlation between the actual fixed aggregate radius and the estimated radii. The GM and SFSE cannot take into account a variation in the aggregate compactness at a large hematocrit, since it is interpreted as a change in the aggregate size.

In the case of the SFSE, one could have expected a fixed value of the estimated radius $R_{g}^{*}$ and a variation of the estimated packing factor $W^{*}$, when the aggregate radius was fixed and the aggregate compactness varied. However, both $R_{g}^{*}$ and $W^{*}$ increased as the true aggregate compactness was raised. The estimated parameters $R_{g}^{*}$ and $W^{*}$ followed linear relations for all hematocrits (see Fig. 4b), as observed previously in Fig. 2b when the aggregate radius $r_{a g} / a$ was changed. It means that $W^{*}$ and $R_{g}^{*}$ carry the same information and that the BSC parametrization is reduced to one parameter.

The estimated parameters using the EMTSFM presented in Fig. 6 show that the model gave quantitatively satisfactory estimates for all aggregate compactnesses and for all studied hematocrits. Contrary to the GM and SFSE, the EMTSFM provided a quasi-constant aggregate radii between 5.7 and 5.9 for both studied hematocrits. Moreover, the aggregate compactnesses were estimated with relative errors less than $12 \%$ at both studied hematocrits for that model. The errors between simulated $B S C_{S F M}$ and the fitted curves were also smaller with the EMTSFM, as it can be observed in Fig. 10b. To conclude, the EMTSFM was the model that explained better the simulated $B S C_{S F M}$.

\section{Computation of $R B C$ distributions and of $B S C_{S F M}$}

The two methods we used here to obtain the RBC spatial distributions did not take into consideration realistic interactions between RBCs. These methods were already presented in Ref. [12] and [11] for the 2D and 3D computer simulations, respectively. They were simple and fast methods to generate samples containing non-overlapping, identical and isotropic aggregates. The 3D computer simulations allowed to better mimic real data but they are time consuming (see section III.A and section V of Ref. [11] to obtain a quick review of different approaches to simulate compact RBC aggregates). In order to simulate the BSC data with the SFM reference model, the method we chose to distribute RBCs in the 3D case allowed (1) to study various aggregate sizes with the same aggregate compactness and (2) to reach the physiological hematocrit of $40 \%$ by mixing identical RBC aggregates and disaggregated RBCs. Since studied scattering models assumed that all RBCs were aggregated in blood and since the averaged percentage of disaggregated RBCs was small (around 25\%), the influence of the disaggregated RBCs on the simulated $B S C_{S F M}$ was neglected during the inversion procedure of the 3D BSC data. Contrary to the $3 \mathrm{D}$ modeling, the method we chose to distribute $\mathrm{RBCs}$ in the $2 \mathrm{D}$ case allowed (1) to study the clustering condition where the aggregate compactness vary and the aggregate size is fixed and (2) to have only aggregated RBCs in blood. However, the 2D computer simulations were limited to a maximum hematocrit of $30 \%$ because of 
the difficulty to simulate with the SFM values greater than $30 \%$. To clarify, the main difficulty in the 2D case was to distribute compact aggregates and to have only aggregated RBCs in blood. The maximum value of the aggregate area fraction $\phi_{a g_{\max }}$ was fixed to 0.5 , corresponding to the maximum particle area fraction that can be easily generated using a random number generator. The procedure we chose to distribute the RBCs within aggregates allows reaching a maximum value of aggregate compactness $\phi_{i_{\max }}$ equal to 0.6 (see section III.B. in Ref. [12]). As a consequence, the maximum value of the systemic hematocrit was limited to: $\phi_{\max }=\phi_{a g_{\max }} \phi_{i_{\max }}=0.3$.

We also modeled individual biconcave RBCs as spheres of equivalent volume in the 3D study and studied BSC between 4 and $45 \mathrm{MHz}$. The impact of modeling a RBC by a sphere on the frequency dependence of the backscatter cross-section has been studied and errors are introduced above $20 \mathrm{MHz}$ [16] [25]. The impact of this simplification on the simulated $B S C_{S F M}$ and structural aggregate estimates with the three models (SFSE, GM and EMTSFM) is unknown and still needs to be explored.

\section{On the use of the EMTSFM in vivo}

The EMTSFM assumes that all RBCs are aggregated in blood and that aggregates are identical and isotropic. Therefore, the BSC behavior obtained in our simulations have pronounced frequency peaks. In experimental conditions [10], the BSC behavior was smoother and the peaks were less pronounced. The reason behind this might be that real blood contains several sizes of aggregates, and since the location of BSC peaks are different for different aggregate populations, a relatively smoother BSC curve can be obtained. Another important aspect to consider is the assumption of isotropic aggregates. In human blood, low shear rates can promote the formation of RBC aggregates having anisotropic (i.e. rouleaux) or isotropic (i.e. clump) structures. The rouleaux like pattern is typically associated to normal blood. However, as the binding energy between RBCs increases with inflammation [26], aggregates form clump structures such as in diabetes mellitus [14] [15]. The assumption of isotropic aggregates in the EMTSFM is thus valid as far as we are concerned with the study of pathological states. In the case of normal human rouleaux of RBCs, if the EMTSFM is applied to estimate structural parameters such as the aggregate size and compactness, this assumption would obviously create a bias against the parameter estimation. Therefore, future improvements should consider incorporating the aggregate anisotropy and the polydispersity in terms of aggregate size and compactness to provide an optimal model for the inversion of experimental data. Future validations will also evaluate the EMTSFM in a controlled Couette flow experiment with ghost RBCs (i.e., optically visible RBCs with no hemoglobin and viable membrane properties) coated with dextran polymers to change attractive energies between erythrocytes and thus modulate the aggregate compactness and size.

Another difficulty to apply the EMTSFM in vivo is that the spectral content of backscattered echoes is also affected by attenuation caused by intervening tissue layers (such as the skin) between the probe and the blood flow. To evaluate correctly microstructural parameters, it is thus of major interest to take into account tissue attenuation effects. Note that the SFSE was slightly modified to introduce the attenuation term in the BSC expression and was named the Structure Factor Size and Attenuation Estimator (SFSAE) [27]. The SFSAE allows to determine simultaneously 
blood structural parameters (i.e., $W^{*}$ and $R_{g}^{*}$ ) and the total attenuation [28] [29]. Future improvements of the EMTSFM should incorporate the tissue attenuation as for the SFSAE. It means that the EMTSFM should be slightly modified by introducing the attenuation term to estimate simultaneously the RBC aggregate size, compactness and the total attenuation.

\section{APPENDIX A}

\section{NUMERICAL COMPUTATION OF THE STRUCTURE FACTOR $S_{a g}$ FOR HARD-CYLINDERS IN 2D}

Since there is no analytical expression of the structure factor $S_{a g}$ for hard-cylinders in 2D [30] [31], $S_{a g}$ was numerically computed for several values $\phi_{a g}$ varying from 0.01 to 0.5 with a step of 0.01 . It means that, in the $2 \mathrm{D}$ case, the cylinder concentration $\phi_{a g}$ was rounded to the second decimal for the computation of $S_{a g}$ in Eq. (7). Note that the computation of $S_{a g}$ depends not only on the area fraction $\phi_{a g}$ but also on the effective particle radius $r_{a g}$. That is why $S_{a g}$ that depends on $r_{a g}$ was computed in a dimensionless way as described next.

For each specified value of $\phi_{a g}$, aggregates of an arbitrarily normalized (dimensionless) radius of 1/60 were randomly distributed within a dimensionless surface area $L^{\prime 2}=1 \times 1$ using a random number generator with nonoverlapping positions. The corresponding density matrix $D^{\prime}$ was computed by dividing the square simulation plane $L^{\prime 2}$ in $N_{p}^{2}$ pixels (herein, $N_{p}=512$ ) and by counting the number of particles falling into each pixel. The Fourier transformation of the density matrix $D^{\prime}$ was then computed to generate a structure factor $S_{a g}$. A mean $S_{a g}$ was determined by repeating 400 times this procedure. When the value of the effective particle radius $r_{a g}$ was specified, the centered grid of wavevectors for the structure factor $S_{a g}$ was computed between $\pm \frac{\pi N_{p}}{2 \times 60 r_{a g}}$ with a step of $\Delta k=\frac{\pi}{60 r_{a g}}$ (i.e., by putting the simulated surface length $L^{\prime}=60 r_{a g}$ ).

\section{APPENDIX B}

ANALYTICAL EXPRESSION OF THE STRUCTURE FACTOR $S_{a g}$ FOR HARD-SPHERES IN 3D

The structure factor $S$ for hard-spheres is given by [23] [32]

$$
S(k)=\frac{1}{1-4 \pi m d^{3} \int_{0}^{1} z^{2} \frac{\sin (2 k z)}{2 k z} c(z) d z}
$$

where $m$ is the number density of hard-spheres, $d$ is the hard-sphere diameter and $c(z)$ is the direct correlation function given by [23] [32]:

$$
\begin{aligned}
-c(z) & =c_{0}+c_{1} \frac{z}{d}+c_{3}\left(\frac{z}{d}\right)^{3} & & \text { for } z \leq d \\
& =0 & & \text { for } z>d
\end{aligned}
$$

The coefficients $c_{0}, c_{1}$ and $c_{3}$ are given by [23] [32]

$$
\begin{aligned}
& c_{0}=\frac{(1+2 \phi)^{2}}{(1-\phi)^{4}} \\
& c_{1}=-\frac{6 \phi(1+\phi / 2)^{2}}{(1-\phi)^{4}} \\
& c_{3}=\frac{\phi}{2} c_{0}=\frac{\phi(1+2 \phi)^{2}}{2(1-\phi)^{4}}
\end{aligned}
$$




\section{ACKNOWLEDGMENTS}

This work was supported by the Fonds Incitatifs Recherche of the Université de Provence and by an operating grant from the Canadian Institutes of Health Research ( $\sharp$ MOP-84358).

\section{REFERENCES}

[1] F. L. Lizzi, M. Ostromogilsky, E. J. Feleppa, M. C. Rorke, and M. M. Yaremko, "Relationship of ultrasonic spectral parameters to features of tissue microstructure", IEEE Trans. Ultrason. Ferroelect. Freq. Contr., vol. 33, pp. 319-329, 1986.

[2] M. F. Insana, R. F. Wagner, D. G. Brown and T. J. Hall, "Describing small-scale structure in random media using pulse-echo ultrasound", J. Acoust. Soc. Am., vol. 87, no. 1, pp. 179-192, 1990.

[3] E. J. Feleppa, F. L. Lizzi, D. J. Coleman, and M. M. Yaremko, "Diagnostic spectrum analysis in ophthalmology: a physical perspective", Ultrasound Med. Biol., vol. 12, pp. 623-631, 1986.

[4] E. J. Feleppa, T. Liu, A. Kalisz, M. C. Shao, N. Fleshner, and V. Reuter, "Ultrasonic spectral-parameter imaging of the prostate", Int. J. Imag. Syst. Technol., vol. 8, pp. 11-25, 1997.

[5] M. L. Oelze, W. D. O’Brien, J. P. Blue, and J. F. Zachary, "Differentiation and characterization of rat mammary fibroadenomas and 4T1 mouse carcinomas using quantitative ultrasound imaging”, IEEE Trans. Med. Imaging, vol. 23, pp. 764-771, 2004.

[6] H. Kitamura, B. Sigel, J. Machi, E. J. Feleppa, J. Sokil-Melgar and J. Justin, "Roles of hematocrit and fibrinogen in red cell aggregation determined by ultrasonic scattering properties", Ultrasound Med. Biol., vol. 21, pp. 827-832, 1995.

[7] R. B. Ami, G. Barshtein, D. Zeltser, Y. Goldberg, I. Shapira, A. Roth, G. Keren, H. Miller, V. Prochorov, A. Eldor, S. Berliner and S. Yedgar, "Parameters of red blood cell aggregation as correlates of the inflammatory state", Am. J. Physiol. Heart Circ. Physiol., vol. 280, pp. H1982-H1988, 2001.

[8] D. Savery and G. Cloutier, "A point process approach to assess the frequency dependence of ultrasound backscattering by aggregating red blood cells”, J. Acoust. Soc. Am., vol. 110, no. 6, pp. 3252-3262, 2001.

[9] I. Fontaine, D. Savery and G. Cloutier, "Simulation of ultrasound backscattering by red blood cell aggregates: effect of shear rate and anisotropy, Biophysical Journal, vol. 82, pp. 1696-1710, 2002.

[10] F. T. H. Yu and G. Cloutier, "Experimental ultrasound characterization of red blood cell aggregation using the structure factor size estimator", J. Acoust. Soc. Am., vol. 122, no. 1, pp. 645-656, 2007.

[11] R. K. Saha, E. Franceschini and G. Cloutier, "Assessment of accuracy of the structure-factor-size-estimator method in determining red blood cell aggregate size from ultrasound spectrum backscattering coefficient”, J. Acoust. Soc. Am., vol. 129, no. 4, pp. $2269-2277,2011$.

[12] Franceschini E., Metzger B. and Cloutier G., "Forward problem study of an effective medium model for ultrasound blood characterization", IEEE Trans. ultras. Ferroelectr. Freq. Control., vol. 58, no. 12, pp. 2668-2679, 2011.

[13] "Handbook of hemorheology and hemodynamics", edited by O. K. Baskurt, M. R. Hardeman, M. W. Rampling and H. J. Meiselman (IOS Press, Amsterdam, Berlin, Oxford, Tokyo, Washington DC, 2007)

[14] H. Schmid-Schonbein, H. Malotta and F. Striesow, "Erythrocyte aggregation: causes, consequences and methods of assessment", Tijdschr NVKS, vol. 15, pp. 88-97, 1990.

[15] H. Schmid-Schonbein, G. Gallasch, J. V. Gosen, E. Volger and H. J. Klose, "Red cell aggregation in blood flow. I. New methods of quantification”, Klin. Wschr., vol. 54, pp. 149-157, 1976.

[16] D. Savery and G. Cloutier, "High-frequency ultrasound backscattering by blood: Analytical and semi-analytical models of the erythrocyte cross section", J. Acoust. Soc. Am., vol. 23, no. 4, pp. 3963-3971, 2007.

[17] P. M. Morse and K. U. Ingard, "Theoretical Acoustics" (Princeton University Press, Princeton, NJ, 1968), Chap. 8, pp. $400-466$.

[18] M. F. Insana and D. G. Brown, "Acoustic scattering theory applied to soft biological tissues", in Ultrasonic Scattering in Biological Tissues, edited by K. K. Shung and G. A. Thieme (CRC, Boca Raton, FL, 1993), Chap.4, pp. 76-124.

[19] I. Fontaine, M. Bertrand and G. Cloutier, "A system-based approach to modeling the ultrasound signal backscattered by red blood cells", Biophysical Journal, vol. 77, pp. 2387-2399, 1999.

[20] V. Twersky, "Low-frequency scattering by correlated distributions of randomly oriented particles", J. Acoust. Soc. Am., vol. 81, no. 5, pp. 1609-1618, 1987. 
[21] K. K. Shung, "On the ultrasound scattering from blood as a function of hematocrit", IEEE Trans. Sonics Ultrason. SU-29, pp. 327-331, 1982.

[22] A. Guinier and J. Fournet, "Small angle scattering of X-rays" (Wiley Interscience, New York 1955), Chap XIV, pp. 637-669.

[23] M. S. Wertheim, "Exact solution of the Percus-Yevick integral equation for hard spheres", Physical Review Letters, vol. 10, no. 8, pp. 321-323, 1963.

[24] E. Franceschini and R. Guillermin, "Experimental assessment of four ultrasound scattering models for characterizing concentrated tissuemimicking phantoms”, J. Acoust. Soc. Amer., vol. 132, pp. 3735-3747, 2012.

[25] C. C. Coussios, "The significance of shape and orientation in single-particle weak-scatterer models", J. Acoust. Soc. Am., vol. 112, pp. 906-915, 2002.

[26] X. Weng, G. Cloutier, R. Beaulieu, G. O. Roederer, "Influence of acute-phase proteins on erythrocyte aggregation", Am. J. Physiol., vol. 271, pp. H2346-H2352, 1996.

[27] E. Franceschini, F. T. H. Yu, F. Destrempes and G. Cloutier, "Ultrasound characterization of red blood cell aggregation with intervening attenuating tissue-mimicking phantoms”, J. Acoust. Soc. Am., vol. 127, pp. 1104-1115, 2010.

[28] F. T. H. Yu, J. K. Armstrong, J. Tripette, H. J. Meiselman and G. Cloutier, "A local increase in red blood cell aggregation can trigger deep vein thrombosis: Evidence based on quantitative cellular ultrasound imaging”, J. Thrombosis and Haemostasis, vol. 9, pp. 481-488, 2011.

[29] J. Tripette, A. Y. Denault, L. Allard, B. Chayer, L. P. Perrault and G. Cloutier, "Ultrasound monitoring of RBC aggregation as a real-time marker of the inflammatory response in a cardiopulmonary bypass swine model", Critical Care Medicine, vol. 41, no. 8, pp. e171-e178, 2013.

[30] C. F. Tejero and J. A. Cuesta, "Hard-sphere and hard-disk freezing from the differential formulation of the generalized effective liquid approximation”, Physical Review E, vol. 47, no. 1, pp. 491-496, 1993.

[31] X. Guo and U. Riebel, "Theoretical direct correlation function for two-dimensional fluids of monodisperse hard spheres", The Journal of Chemical Physics, vol. 125, 144504, 2006.

[32] N. W. Ashcroft, "Structure and resistivity of liquid metals", Physical Review, vol. 145, pp. 83-90, 1966. 


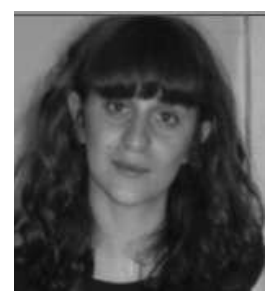

Emilie Franceschini received her M.S. degree in Mechanical Engineering from the Ecole Supérieure d'Ingénieurs de Marseille in 2003, and the Ph.D. degree in Acoustics from the University of Provence, Marseille, 2006. In 2007, she was a Postdoctoral Fellow at the Laboratory of Biorheology and Medical Ultrasonics, Research Center of the University of Montreal Hospital, Montreal, QC. Since October 2008, she is a Researcher at the French National Centre for Scientific Research (CNRS) in the Laboratory of Mechanics and Acoustics CNRS - UPR 7051, Marseille. Her current research interests include ultrasound imaging for biomedical applications, inverse problems and ultrasound characterization of biological tissues at the microscopic level. She is a member of the French Acoustical Society (SFA) and IEEE.

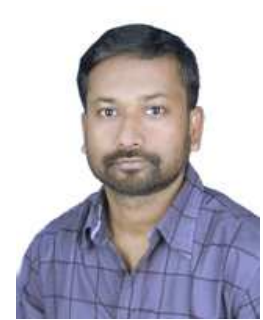

Ratan K. Saha obtained his B. Sc. in Physics (studied at A B N Seal College, Coochbehar, India) from the University of North Bengal, Siliguri, India in 1996 and received his M. Sc. in Physics from Jadavpur University, Kolkata, India in 1999. He carried out his Ph. D. work at the Saha Institute of Nuclear Physics, Kolkata during 2000-06. After that he joined the Laboratory of Biorheology and Medical Ultrasonics, Research Center of the University of Montreal Hospital, Montreal, Canada as a post-doctoral fellow (2007-08). He also worked as a post-doctoral fellow at Ryerson University, Toronto, Canada (2009-11). Since October 2011, he is a post-doctoral fellow at Saha Institute of Nuclear Physics and his current research interests include ultrasonic and photoacoustic characterizations of soft tissues.

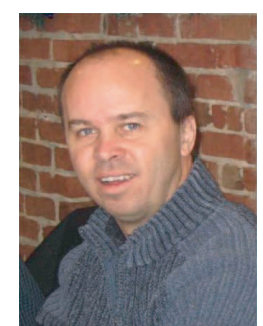

Guy Cloutier (S`89-M`90-SM`07) obtained his B.Eng. in Electrical Engineering (84), and M.Sc. and Ph.D. in Biomedical Engineering $(86,90)$. Between the years 90-92, he was a post-doctoral fellow at The Pennsylvania State University with Prof. K. Kirk Shung. Prof. Cloutier is Director of the Laboratory of Biorheology and Medical Ultrasonics at the University of Montreal Hospital Research Center (www.lbum-crchum.com), and Professor of Radiology and Biomedical Engineering at the University of Montreal. His research interests are in quantitative ultrasound imaging of red blood cell aggregation, quasi-static and dynamic ultrasound elastography of atherosclerotic plaques, vascular aneurysms, deep vein thrombi and breast cancers, 3D morphologic and hemodynamic assessment of lower limb arterial stenoses, and mathematical and biomechanical modeling. He has published more than 130 peer-reviewed articles in these fields, holds 11 patents, and was recipient of the National Scientist award of the Fonds de Recherche en Santé du Québec (2004-2009). 


\section{TABLE CAPTION}

Table I. Acoustical properties of blood found in [16] and [21].

Table II. Values of the aggregate radius and compactness used for computation of the simulated $B S C_{S F M}$, and values of parameters found with the SFSE. Aggregating conditions: $r_{a g} / a$ varies, $\phi$ varies, $\phi_{i}=60 \%$ (except in the case of diaggregated RBCs where $\phi_{i}=100 \%$ ). The parameter $\epsilon$ indicates the relative error. 
TABLE I

\begin{tabular}{lccc}
\hline & $\begin{array}{c}\text { Density } \\
\rho\left(\mathrm{kg} . \mathrm{m}^{-3}\right)\end{array}$ & $\begin{array}{c}\text { Compressibility } \\
\kappa\left(\mathrm{Pa}^{-1}\right)\end{array}$ & $\begin{array}{c}\text { Impedance } \\
Z \text { (MRayl) }\end{array}$ \\
\hline RBC & 1092 & $3.41 \times 10^{-10}$ & 1.766 \\
\hline Plasma & 1021 & $4.09 \times 10^{-10}$ & 1.580 \\
\hline
\end{tabular}


TABLE II

\begin{tabular}{|c|c||c|c|c||c|c|c||c|c|c||}
\hline \multicolumn{2}{|c|}{ SFM } & \multicolumn{3}{c|}{$\phi=10 \%$} & \multicolumn{3}{c|}{$\phi=20 \%$} & \multicolumn{3}{|c|}{$\phi=30 \%$} \\
\hline$r_{a g} / a$ & $\begin{array}{c}\phi_{i} \\
(\%)\end{array}$ & $W^{*}$ & $R_{g}^{*}$ & $\begin{array}{c}\epsilon_{R_{g}^{*}} \\
(\%)\end{array}$ & $W^{*}$ & $R_{g}^{*}$ & $\begin{array}{c}\epsilon_{R_{g}^{*}} \\
(\%)\end{array}$ & $W^{*}$ & $R_{g}^{*}$ & $\begin{array}{c}\epsilon_{R_{g}^{*}} \\
(\%)\end{array}$ \\
\hline 1 & 100 & 0.61 & 0.39 & -61.00 & 0.37 & 0.39 & -61.00 & 0.17 & 0.38 & -62.00 \\
\hline 3.16 & 60 & 3.12 & 1.50 & -52.53 & 3.29 & 1.56 & -50.63 & 2.67 & 1.32 & -58.23 \\
\hline 5 & 60 & 7.41 & 3.81 & -23.80 & 6.95 & 3.64 & -27.20 & 5.31 & 3.04 & -39.20 \\
\hline 7.07 & 60 & 15.82 & 7.99 & 13.01 & 13.57 & 7.18 & 1.56 & 8.58 & 5.33 & -24.61 \\
\hline
\end{tabular}




\section{FIGURE CAPTIONS}

Figure 1. Frequency-dependent BSCs for different aggregate sizes and a constant aggregate compactness $\phi_{i}=60 \%$ at systemic hematocrits of 10,20 and $30 \%$. The symbols represent the $B S C_{S F M}$ computation. The dashed lines represents the corresponding fitting with the SFSE, whereas the solid lines expresses the fitting with the EMTSFM.

Figure 2. a) Comparison of $R_{g}^{*}$ estimated with SFSE and the actual aggregate size $r_{a g} / a$ for the three systemic hematocrits 10, 20 and 30\%. b) Linear relationships between $W^{*}$ and $R_{g}^{*}$. Results presented here correspond to the configuration where $r_{a g} / a$ varies and $\phi_{i}$ is fixed.

Figure 3. Frequency-dependent BSCs computed with the SFM for different aggregate compactnesses and a constant aggregate size $r_{a g} / a=6.32$ at systemic hematocrits of 10 and $20 \%$, and corresponding fitting with the SFSE (in dashed lines) and with the EMTSFM (in solid lines).

Figure 4. a) Aggregate size $R_{g}^{*}$ estimated with the SFSE as a function of different aggregate compactnesses for systemic hematocrits of 10 and $20 \%$. The solid line represents the actual aggregate size $r_{a g} / a=6.32$. b) Linear relationships between $W^{*}$ and $R_{g}^{*}$. Results presented here correspond to the configuration where $\phi_{i}$ varies and $r_{a g} / a$ is fixed.

Figure 5. a) Values of $r_{a g}^{*} / a$ and $\phi_{i}^{*}$ estimated by the EMTSFM as a function of the actual aggregate radius for the three systemic hematocrits of 10, 20 and 30\%. Also represented are actual values of $r_{a g} / a$ and $\phi_{i}$. (b) Corresponding relative errors of $r_{a g}^{*} / a$ and $\phi_{i}^{*}$.

Figure 6. a) Values of $r_{a g}^{*} / a$ and $\phi_{i}^{*}$ estimated by the EMTSFM as a function of the actual aggregate compactness for the systemic hematocrits of 10 and $20 \%$. Also represented are actual values of $r_{a g} / a$ and $\phi_{i}$.

(b) Corresponding relative errors of $r_{a g}^{*} / a$ and $\phi_{i}^{*}$.

Figure 7. Frequency-dependent BSCs computed with the SFM for different aggregate sizes and a constant aggregate compactness $\phi_{i}=60 \%$ at systemic hematocrits of 10,20 and $30 \%$, and corresponding fitting with the GM.

Figure 8. a) Values of $a_{e f f}^{*} / a$ and $\phi_{i}^{*}$ estimated by the GM as a function of the actual aggregate radius for the three systemic hematocrits of 10,20 and 30\%. Also represented are actual values of $r_{a g} / a$ and $\phi_{i}$. (b) Corresponding relative errors of $a_{\text {eff }}^{*} / a$ and $\phi_{i}^{*}$.

Figure 9. a) Values of $a_{e f f}^{*} / a$ and $\phi_{i}^{*}$ estimated by the GM as a function of the actual aggregate compactness for the systemic hematocrits of 10 and $20 \%$. Also represented are actual values of $r_{a g} / a$ and $\phi_{i}$. (b) Corresponding relative errors of $a_{e f f}^{*} / a$ and $\phi_{i}^{*}$.

Figure 10. Logarithm of the error between the simulated $B S C_{S F M}$ and the fitted curves with the three scattering models GM, SFSE and EMTSFM. a) As a function of the actual aggregate size for the clustering configuration where $r_{a g} / a$ varies and $\phi_{i}$ is fixed. b) As a function of the actual aggregate compactness for the clustering configuration where $\phi_{i}$ varies and $r_{a g} / a$ is fixed. 
Figure 11. a) Concentration of aggregated RBCs $\phi^{\prime}$ as a function of the mean aggregate radius $r_{a g} / a$ for the three systemic hematocrits of 20,30 and 40\%. b) and c) Frequency-dependent BSCs computed with the SFM in the $3 \mathrm{D}$ case for different aggregate sizes and a constant aggregate compactness $\phi_{i}=74 \%$ at systemic hematocrits of 30 and $40 \%$, and corresponding fitting with the SFSE model, the EMTSFM and the GM.

Figure 12. a) Values of $r_{a g}^{*} / a$ and $\phi_{i}^{*}$ estimated by the EMTSFM as a function of the actual aggregate radius for the three systemic hematocrits of 20,30 and 40\%. Also represented are actual values of $r_{a g} / a$ and $\phi_{i}$. (b) Corresponding relative errors of $r_{a g}^{*} / a$ and $\phi_{i}^{*}$.

Figure 13. a) Values of $a_{\text {eff }}^{*} / a$ and $\phi_{i}^{*}$ estimated by the GM as a function of the actual aggregate radius for the three systemic hematocrits of 20,30 and $40 \%$. Also represented are actual values of $r_{a g} / a$ and $\phi_{i}$. (b) Corresponding relative errors of $a_{e f f}^{*} / a$ and $\phi_{i}^{*}$. 

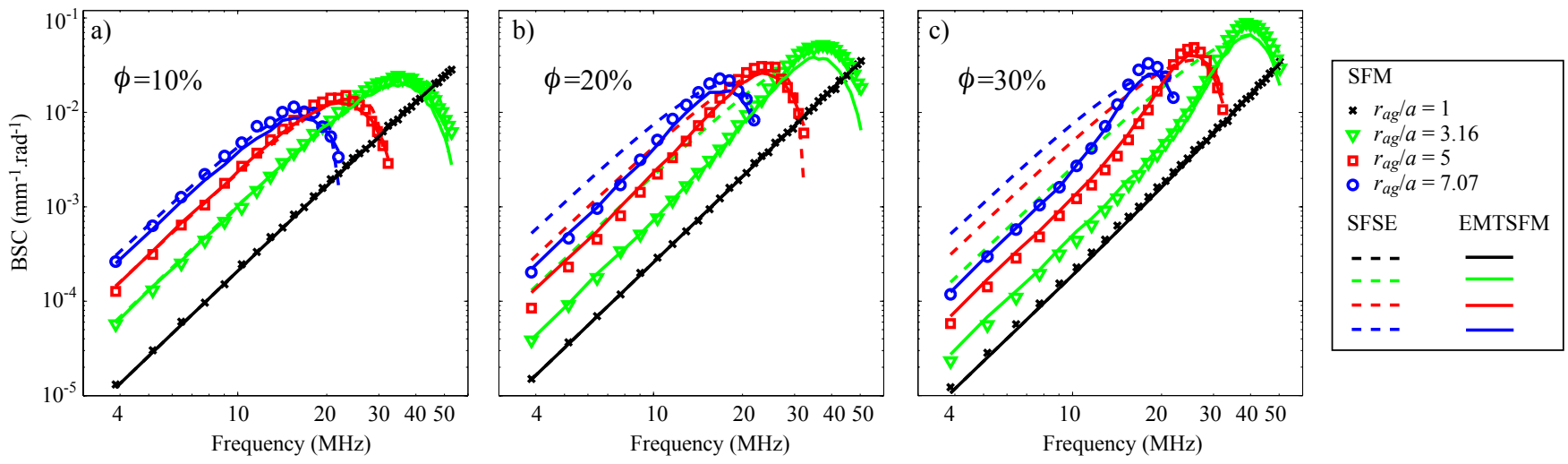

Fig. 1. 

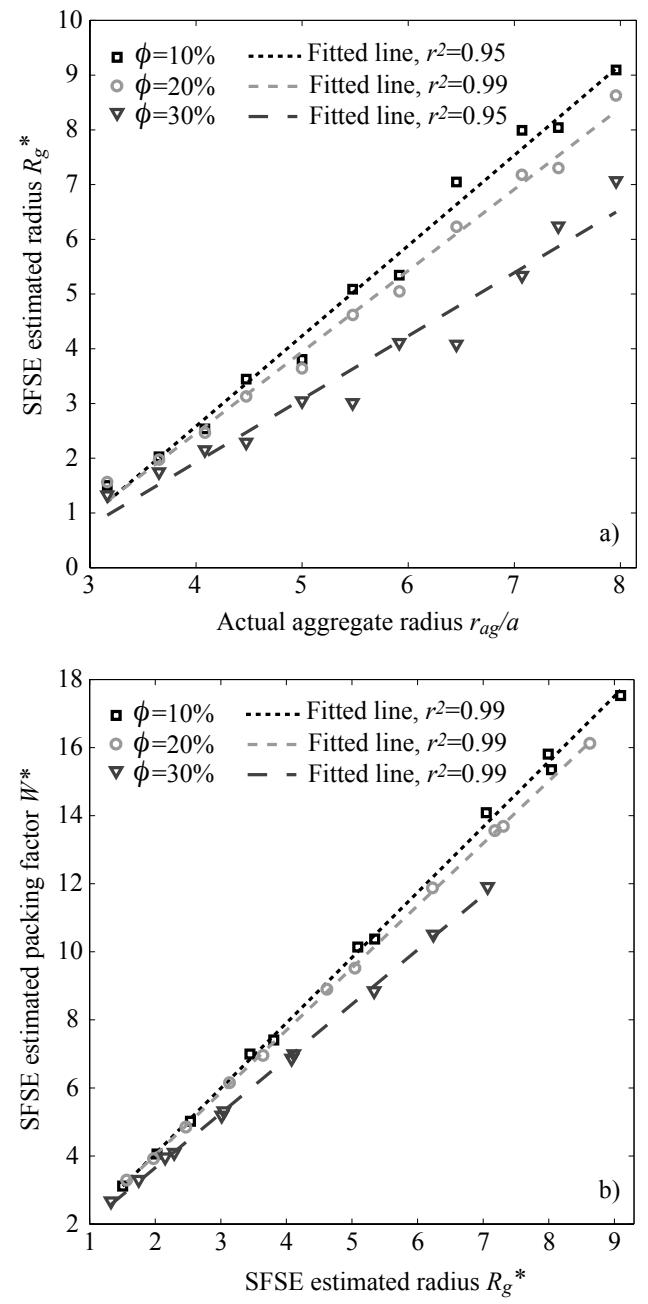

Fig. 2. 

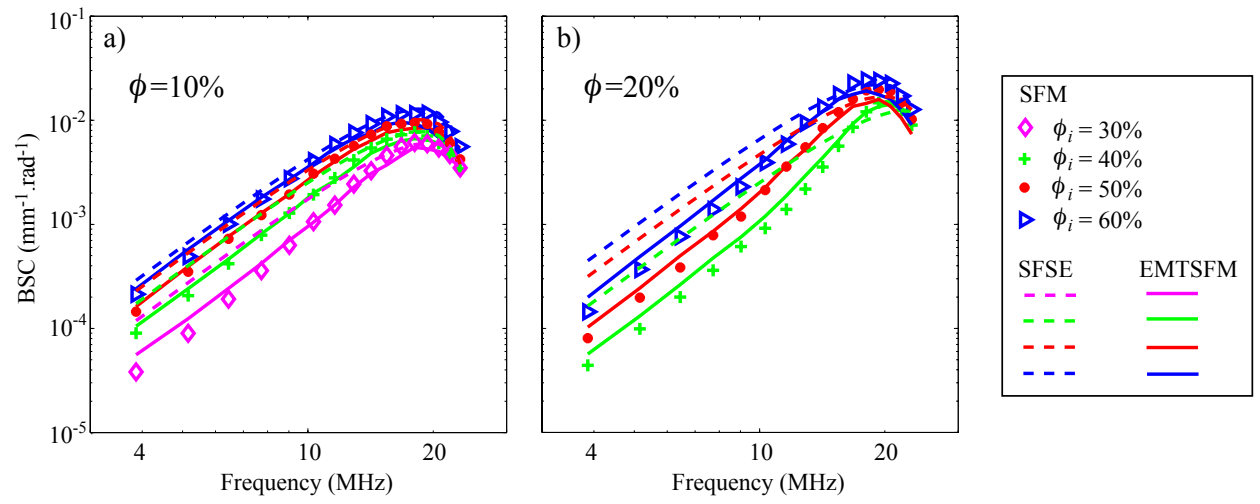

Fig. 3. 

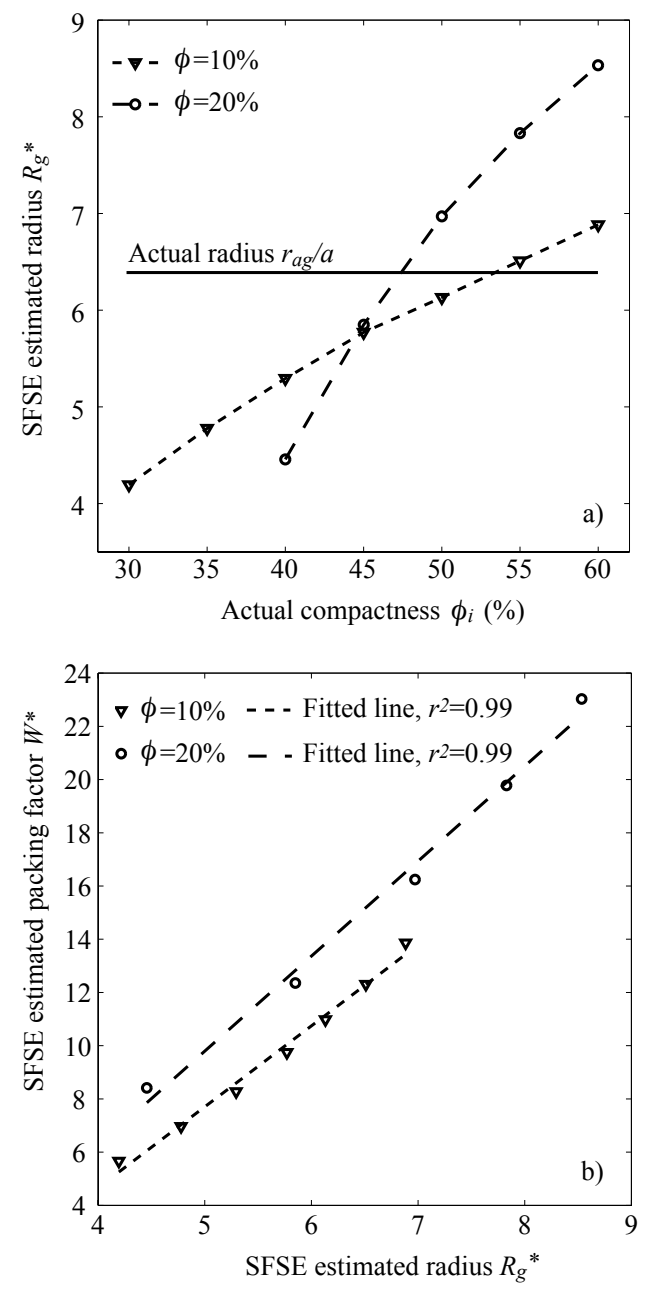

Fig. 4. 

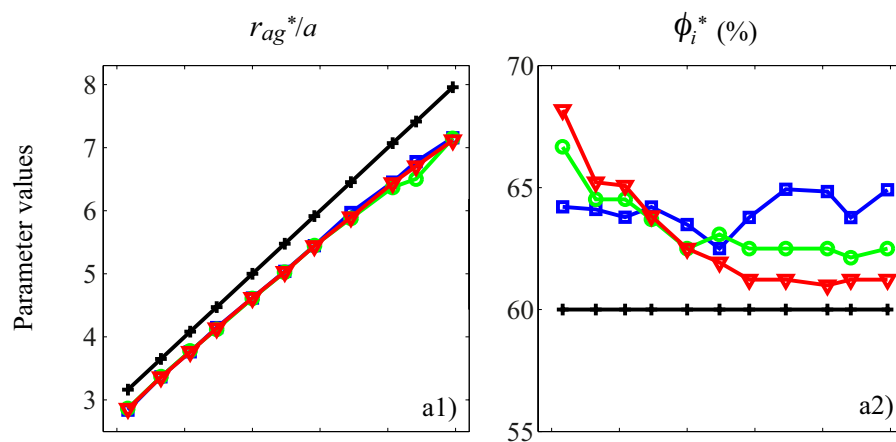

— Actual parameters

$\rightarrow$ EMTSFM $(\phi=10 \%)$
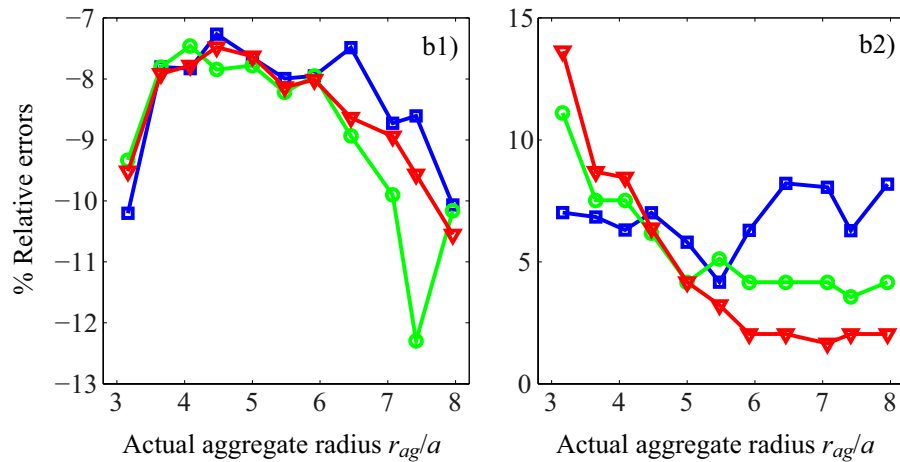

$\multimap \operatorname{EMTSFM}(\phi=20 \%)$

$\neg$ EMTSFM $(\phi=30 \%)$

Fig. 5. 

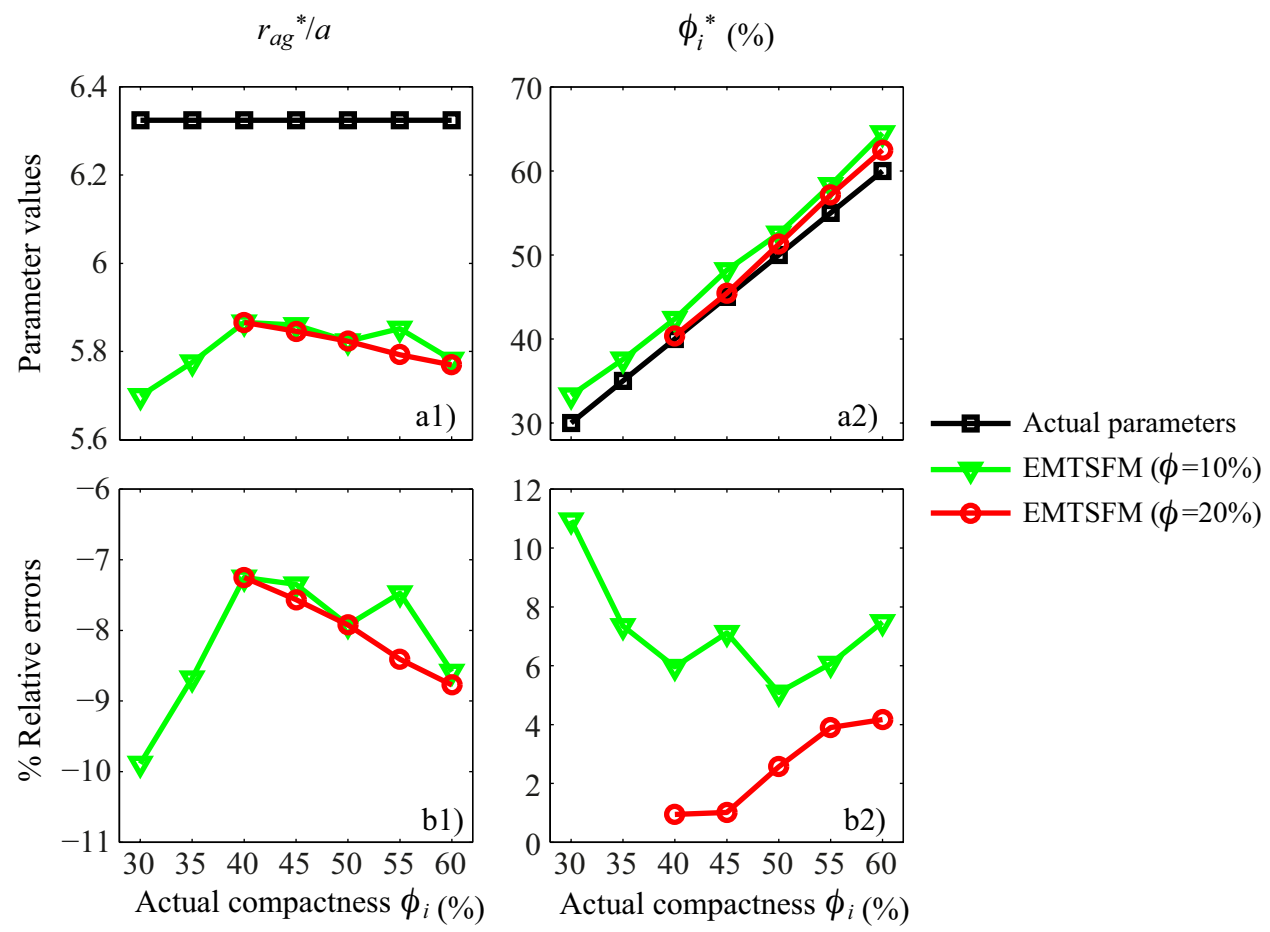

Fig. 6 . 

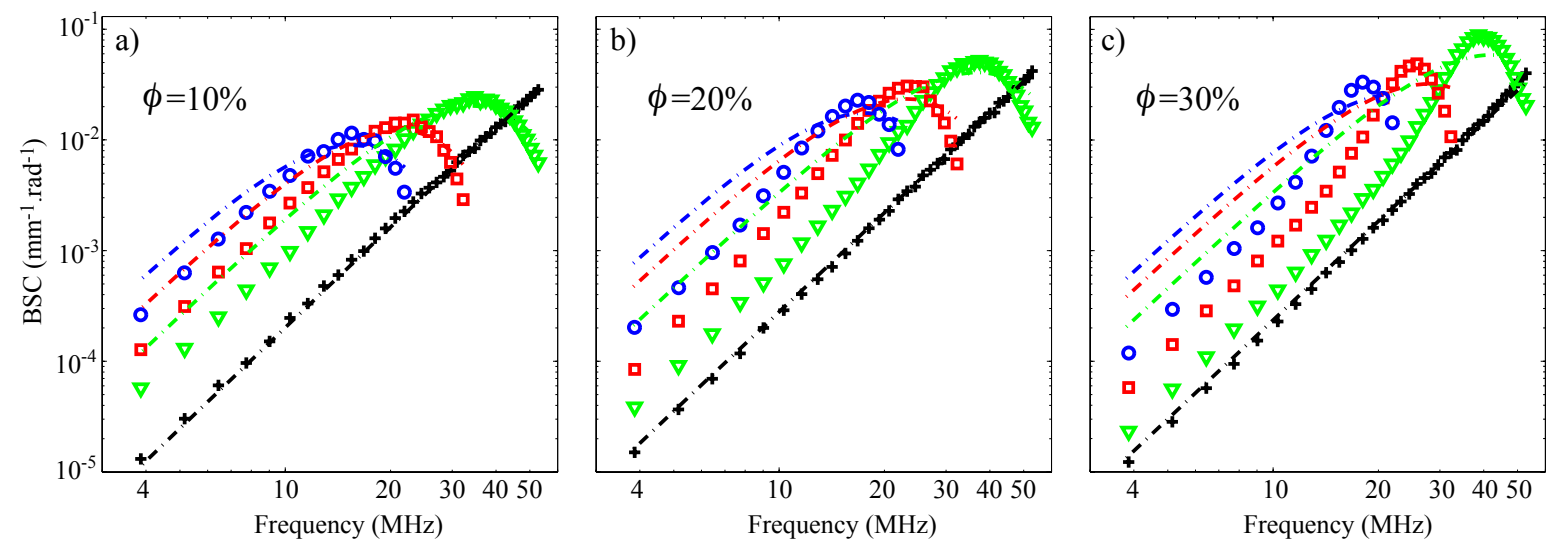

SFM

× $r_{a g} / a=1$

$\nabla r_{a g} / a=3.16$

ㅁ $r_{a g} / a=5$
$0 \quad r_{a g} / a=7.07$

GM

- - - '

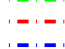

Fig. 7. 

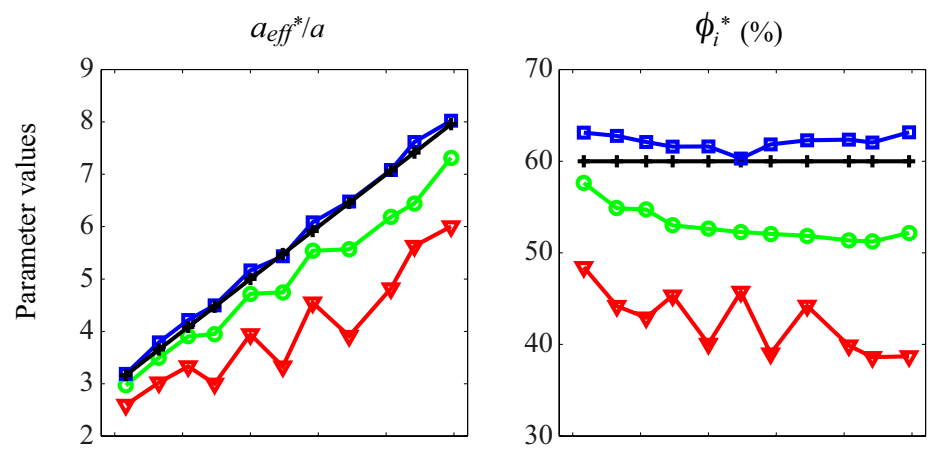

$\longrightarrow$ Actual parameters
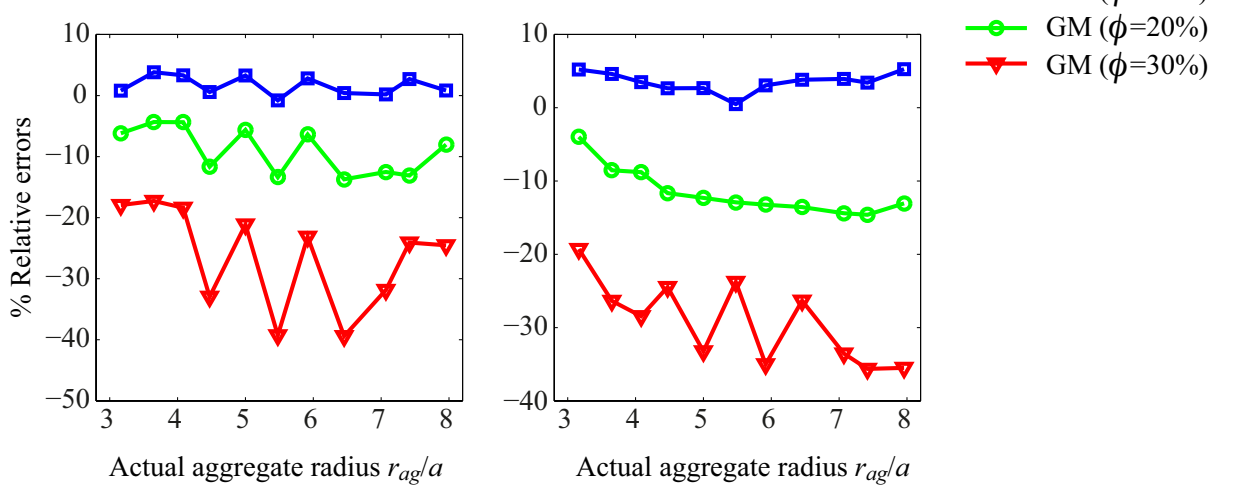

Fig. 8. 

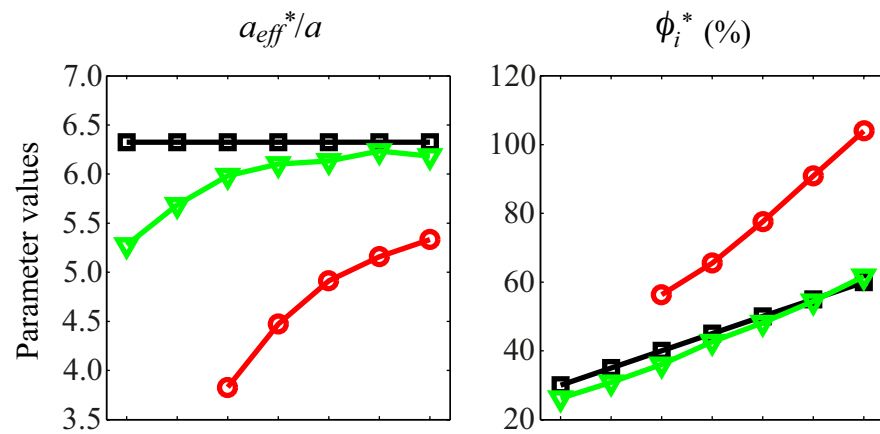

$\boldsymbol{\nabla}$ - Actual parameters
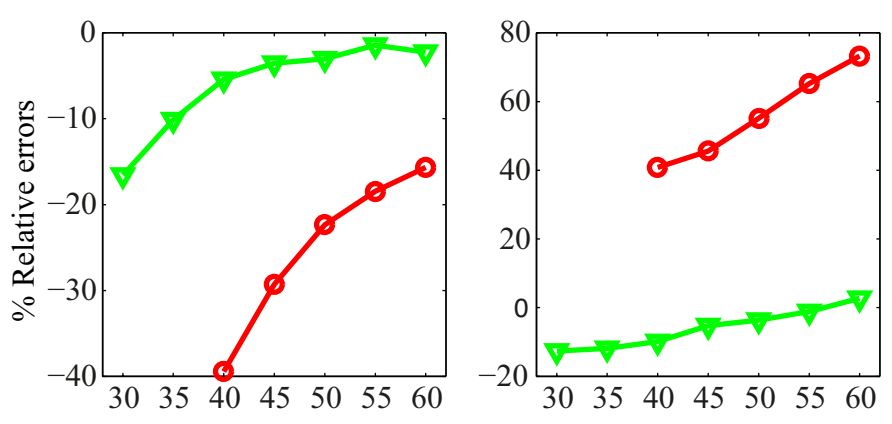

$\because \operatorname{GM}(\phi=10 \%)$

$\multimap \operatorname{GM}(\phi=20 \%)$

Actual compactness $\phi_{i}(\%)$

Actual compactness $\phi_{i}(\%)$

Fig. 9. 

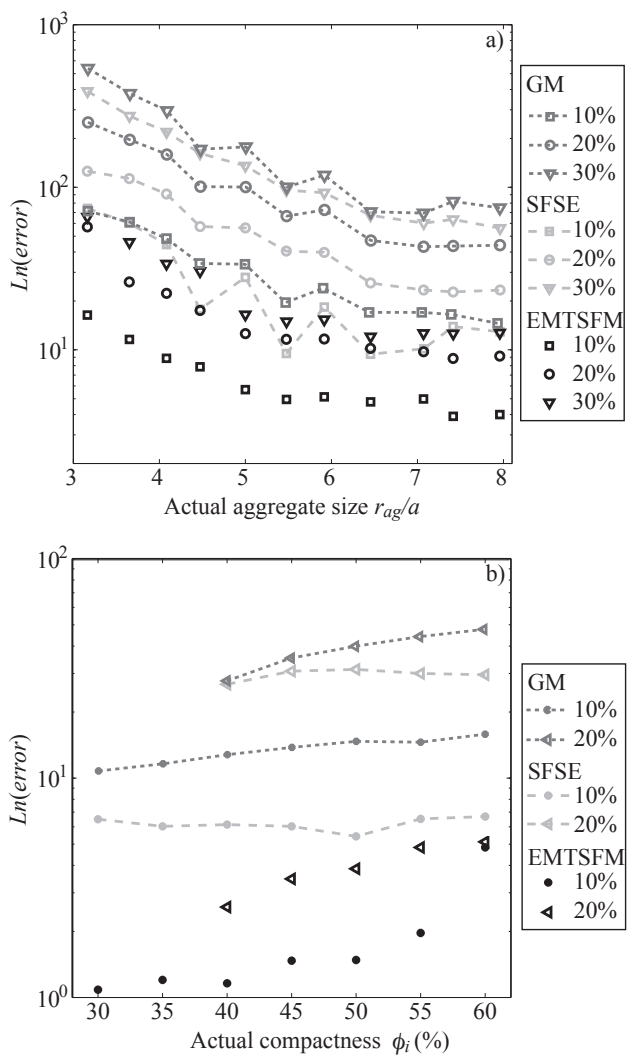

Fig. 10. 

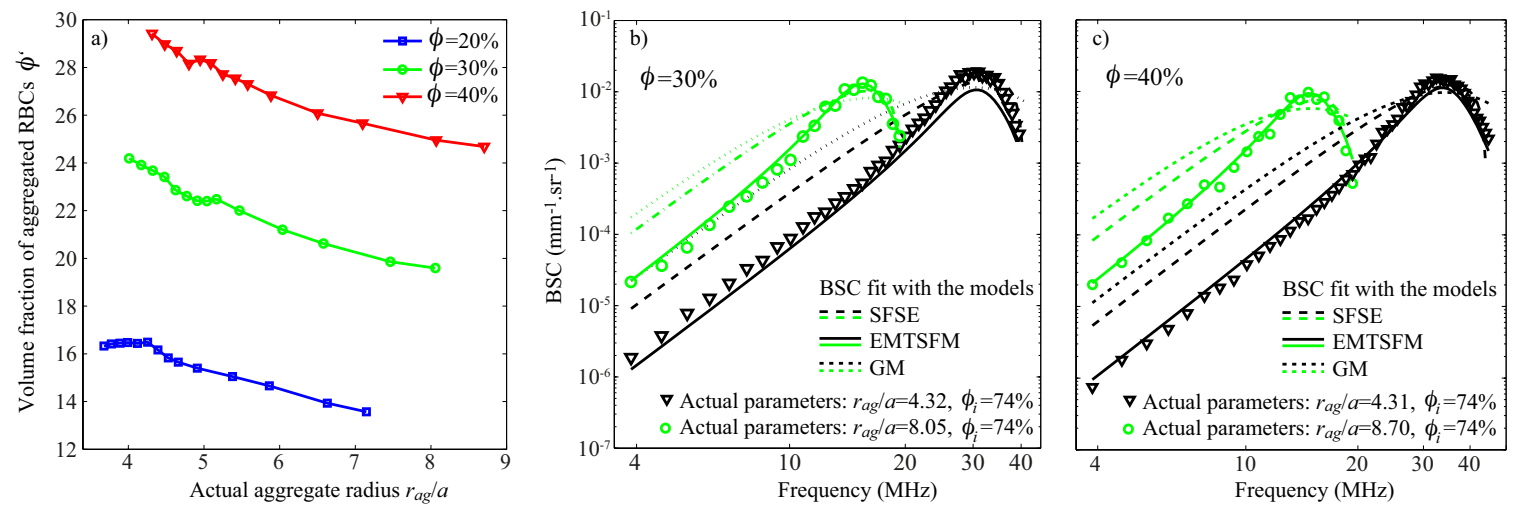

Fig. 11. 

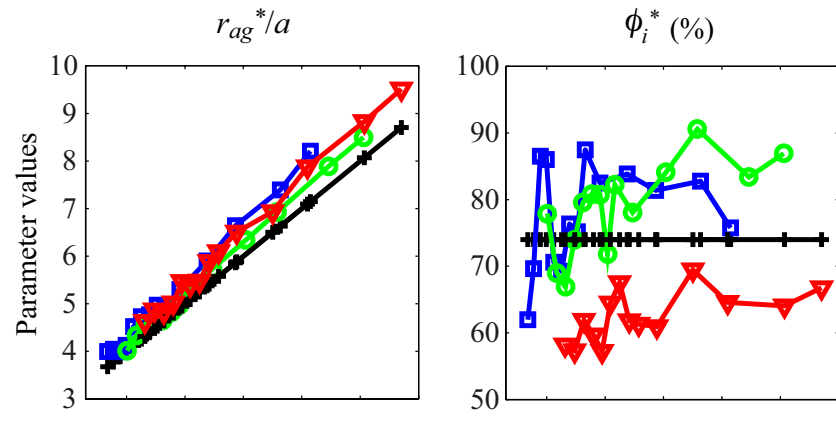

$\longrightarrow$ Actual parameters
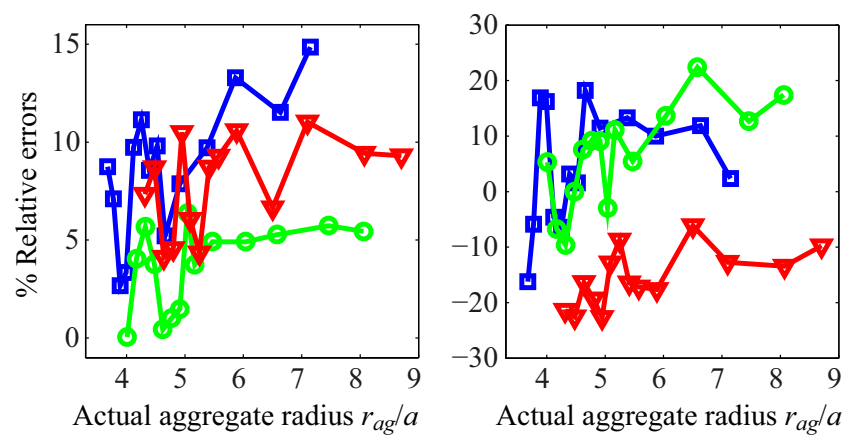

- EMTSFM $(\phi=30 \%)$

$\neg$ EMTSFM $(\phi=40 \%)$

Fig. 12. 

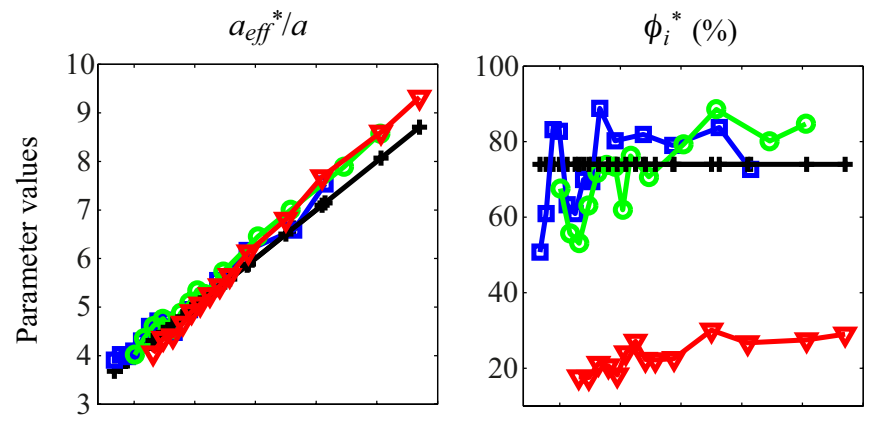

$\longrightarrow$ Actual parameters
$\longrightarrow$ GM $(\phi=20 \%)$
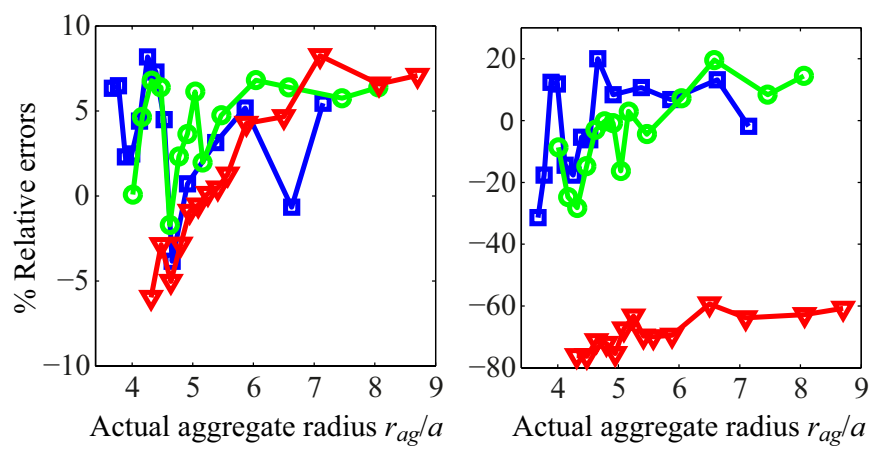

$-\mathrm{GM}(\phi=30 \%)$

$\multimap \operatorname{GM}(\phi=40 \%)$

Fig. 13. 